\title{
Baicalein attenuates the quorum sensing-controlled virulence factors of Pseudomonas aeruginosa and relieves the inflammatory response in $P$. aeruginosa- infected macrophages by downregulating the MAPK and NFKB signal-transduction pathways
}

This article was published in the following Dove Press journal:

Drug Design, Development and Therapy

7 January 2016

Number of times this article has been viewed

\author{
Jing Luo* \\ Jin-liang Kong* \\ Bi-ying Dong \\ Hong Huang \\ Ke Wang \\ Li-hong Wu \\ Chang-chun Hou \\ Yue Liang \\ Bing Li \\ Yi-qiang Chen
}

Department of Respiratory Disease, First Affiliated Hospital of Guangxi Medical University, Nanning, People's Republic of China

*These authors contributed equally to this work

\begin{abstract}
Burgeoning antibiotic resistance and unfavorable outcomes of inflammatory injury after Pseudomonas aeruginosa infection have necessitated the development of novel agents that not only target quorum sensing (QS) but also combat inflammatory injury with the least risk of resistance. This study aimed to assess the anti-QS and anti-inflammatory activities of baicalein, a traditional herbal medicine that is widely used in the People's Republic of China, against $P$. aeruginosa infection. We found that subminimum inhibitory concentrations of baicalein efficiently interfered with the QS-signaling pathway of $P$. aeruginosa via downregulation of the transcription of QS-regulated genes and the translation of QS-signaling molecules. This interference resulted in the global attenuation of QS-controlled virulence factors, such as motility and biofilm formation, and the secretion into the culture supernatant of extracellular virulence factors, including pyocyanin, LasA protease, LasB elastase, and rhamnolipids. Moreover, we examined the anti-inflammatory activity of baicalein and its mode of action via a $P$. aeruginosa-infected macrophage model to address its therapeutic effect. Baicalein reduced the $P$. aeruginosa-induced secretion of the inflammatory cytokines IL-1 $\beta$, IL-6, IL-8, and TNF $\alpha$. In addition, baicalein suppressed $P$. aeruginosa-induced activation of the MAPK and NFKB signal-transduction pathways in cocultured macrophages; this may be the mechanism by which baicalein inhibits the production of proinflammatory cytokines. Therefore, our study demonstrates that baicalein represents a potential treatment for $P$. aeruginosa infection because it clearly exhibits both antibacterial and anti-inflammatory activities.
\end{abstract}

Keywords: baicalein, Pseudomonas aeruginosa, quorum sensing, virulence, inflammatory cytokines, signal transduction

\section{Introduction}

Pseudomonas aeruginosa, an opportunistic Gram-negative bacillus, is a frequent cause of hospital-acquired infections, including ventilator-associated pneumonia ${ }^{1}$ and catheter infections in immunocompromised patients. ${ }^{2}$ The progressive deterioration of the lungs due to chronic $P$. aeruginosa infection and the resulting persistent inflammation are currently the main causes of morbidity and mortality in patients with cystic fibrosis. ${ }^{3,4} P$. aeruginosa produces factors associated with intracellular communication and extracellular virulence that are globally regulated by well-defined quorum-sensing (QS) systems that determine bacterial pathogenesis. ${ }^{5}$ This regulatory system relies
Correspondence: Yi-qiang Chen Department of Respiratory Disease, First Affiliated Hospital of Guangxi Medical University, 6 Shuangyong Road, Qingxiu, Nanning, Guangxi 53002I, People's

Republic of China

Tel/fax +86 77I 5350993

Email chenyq2013@sohu.com 
upon small, diffusible signal molecules ( $N$-acyl-L-homoserine lactones [AHLs]) that interact with transcriptional activators to couple gene expression with cell-population density. The QS system is arranged in a hierarchical manner with the las system in the dominant role, positively controlling the activity of the $r h l$ system. ${ }^{6}$ The las system directs the synthesis of $\mathrm{N}$-(3-oxododecanoyl)-L-homoserine lactone (odDHL), whereas the $r h l$ system uses $N$-butanoyl-L-homoserine lactone (BHL) as its signaling molecule. ${ }^{7,8}$ Virulence factors produced by $P$. aeruginosa are modulated through the QS system; for instance, the production of pyocyanin and rhamnolipids is controlled by the $r h l$ system, whereas LasA protease and LasB elastase activities are controlled by the las system. ${ }^{8}$ In addition, type IV pili and flagella, as well as type II and type III secretion systems, involved in the virulence of $P$. aeruginosa are closely related to QS. ${ }^{9} P$. aeruginosa also adopts a biofilm mode of growth, which is regulated by both the las and $r h l$ systems, making the bacteria recalcitrant to various antimicrobial treatments. ${ }^{10}$

Because the treatment of $P$. aeruginosa infection, including the administration of either $\beta$-lactam alone or combination therapy, including colistin or tobramycin with ceftazidime, has become increasingly compromised by high levels of allergy to $\beta$-lactam and the emergence of microbial antibiotic resistance, ${ }^{11}$ there is an urgent need for novel antimicrobial agents that can evade microbial resistance and that have fewer side effects. Alternative strategies, such as using QS-inhibitory agents to reduce virulence and disarming pathogens rather than killing them, may apply milder evolutionary pressure that does not favor the development of resistance, ${ }^{12}$ which may make them an ideal choice for effectively managing $P$. aeruginosa infection. In addition to therapies that focus on pathogens, anti-inflammatory treatments are in great demand, as both the pathogenicity of the pathogen and the host immune response are involved in the persistence of $P$. aeruginosa infection. A prominent early component of the host immune response to pulmonary infection by $P$. aeruginosa is the secretion of various proinflammatory cytokines, recruiting a massive influx of neutrophils to the infection site. Such excessive inflammatory responses are harmful to the host, contributing to severe tissue damage, organ dysfunction, ${ }^{13}$ and generalized hyperpyrexia. ${ }^{14}$ Therefore, anti-inflammatory drugs may slow the progression of chronic infectious diseases by interrupting the vicious cycle of infection and inflammation. ${ }^{15}$ Plant-derived compounds have been widely used for centuries to combat microbial infections and reduce inflammation, and they are believed to be safe for human consumption. ${ }^{16}$ Based on the aforementioned, screening plant-derived compounds for a promising candidate that can attenuate bacterial pathogenesis by interfering with the QS system, thus rendering pathogenic bacteria nonvirulent without affecting their viability, in addition to possessing potential anti-inflammatory effects, would be of great therapeutic value for combating $P$. aeruginosa infection.

Baicalein (Figure 1), a major flavonoid monomer extracted from the roots of Scutellaria baicalensis, has been described as a medicine in the Chinese Pharmacopoeia and is in wide clinical use for the treatment of fever, sore throat, and upper respiratory tract infection. ${ }^{17,18}$ Moreover, baicalein is commercially produced as oral tablets for the treatment of bacteria-induced diarrhea in the People's Republic of China. ${ }^{17}$ In addition to its prominent inhibitory effects on pathogenic bacteria, fungi, and viruses, ${ }^{19,20}$ baicalein has been found to have anti-inflammatory, antioxidant, neuroprotective, and antitumor activities. ${ }^{21-23}$ Interestingly, baicalein has exhibited remarkable synergy with tetracycline and $\beta$-lactams in the treatment of methicillin-resistant Staphylococcus aureus. ${ }^{24}$ A computer-based virtual screening study demonstrated that baicalein can significantly promote proteolysis of the QS-signal receptor TraR in Escherichia coli, inhibit biofilm formation, and show synergistic activity with ampicillin against $P$. aeruginosa. ${ }^{25}$ These results suggest that baicalein may interfere with the QS system in $P$. aeruginosa. Nevertheless, the mechanism by which baicalein interferes with the QS-controlled virulence factors and genes of $P$. aeruginosa, and the effect of baicalein on the inflammatory response caused by $P$. aeruginosa require further investigation. In this study, we report for the first time that baicalein exerts anti-QS activity through significantly attenuating the virulence factors of $P$. aeruginosa and downregulating the transcription of QS-regulated genes and the translation of QS-signaling molecules. Moreover, the anti-inflammatory effect of baicalein is demonstrated in a $P$. aeruginosa-infected macrophage model, and our results suggest that baicalein reduces proinflammatory cytokine production mainly through downregulating the

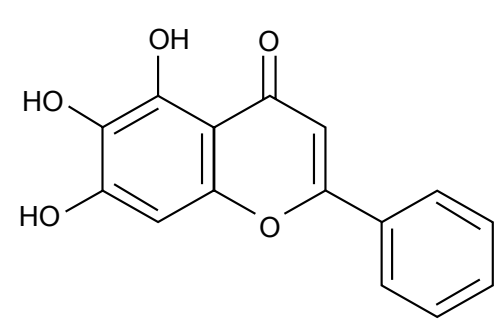

Figure I Chemical structure of baicalein. Note: Molecular weight of baicalein is $270.24 \mathrm{~g} / \mathrm{mol}$. 
MAPK and NFאB signal-transduction pathways. Together, our data suggest that baicalein may be a useful plant-derived compound for the treatment of $P$. aeruginosa infection.

\section{Materials and methods Bacterial strains and reagents}

This study used the sequenced $P$. aeruginosa PAO1 wild-type strain and its mutants deficient in lasR-rhlR ( $\Delta l a s I-\Delta r h l I)$, generously donated by Dr Liang Yang (Nanyang Technological University, Singapore). Strains were routinely stored in Luria-Bertani (LB) broth containing 25\% glycerol at $-80^{\circ} \mathrm{C}$. Baicalein standard dry powder purchased from Sigma-Aldrich (St Louis, MO, USA) with a purity of $\geq 98 \%$ by high-performance liquid chromatography (HPLC) was freshly dissolved in dimethyl sulfoxide (Sigma-Aldrich). The baicalein stock solutions were sterilized by passing through a $0.22 \mu \mathrm{m}$ syringe filter (EMD Millipore, Billerica, MA, USA), and aliquots were stored at $-80^{\circ} \mathrm{C}$ until use.

\section{Determination of minimum inhibitory concentration and growth curve}

The minimum inhibitory concentration (MIC) of baicalein against wild-type $P$. aeruginosa $\mathrm{PAO} 1$ was determined with reference to Clinical and Laboratory Standards Institute standards using a broth-microdilution method with an inoculum of $1 \times 10^{5} \mathrm{CFU} / \mathrm{mL}$ in Müller-Hinton broth as described by Bala et al. ${ }^{26}$ The final tested drug concentrations ranged from 32 to $1,024 \mu \mathrm{g} / \mathrm{mL}$ in serial twofold dilutions. The MIC was defined as the lowest concentration of baicalein that prevented visible growth. For measurements of growth curves, overnight cultures of $P$. aeruginosa inocula were diluted in fresh LB medium to achieve a cell-suspension optical density at $600 \mathrm{~nm}\left(\mathrm{OD}_{600}\right)$ of 0.05 . Then, the suspension was supplemented with appropriate concentrations of baicalein and incubated at $37^{\circ} \mathrm{C}$ under continuous agitation. At the time point indicated, $1 \mathrm{~mL}$ of the mixture was withdrawn; its turbidity was measured at $\mathrm{OD}_{600}$ using a spectrophotometer (Thermo Fisher Scientific, Waltham, MA, USA), and was recorded to generate a growth curve. The sub-MICs were defined as series concentrations equal to or less than the highest concentration of baicalein that did not affect growth based on continuous measurements of cell density. ${ }^{26}$ All experiments were repeated at least three times.

\section{Supernatant or inoculum preparation}

$P$. aeruginosa $\mathrm{PAO} 1$ cultures were grown overnight in LB medium to yield an $\mathrm{OD}_{600}$ of 2.0 and then incubated with appropriate concentrations $(32-128 \mu \mathrm{g} / \mathrm{mL})$ of baicalein for 12 hours or in the absence of agent as a blank control. The $P$. aeruginosa PA01 QS-mutant deficient in lasI-rhlI was grown along with the treated and untreated $P$. aeruginosa PAOl cultures, and it served as a negative control. Cells were harvested by centrifugation and washed twice with sterile phosphate-buffered saline (PBS) for RNA extraction, and the supernatants were sterilized by filtering through a $0.22 \mu \mathrm{m}$ syringe filter (EMD Millipore) before being either used immediately or stored at $-80^{\circ} \mathrm{C}$ for later measurements of pyocyanin, LasA protease, LasB elastase, rhamnolipids, and AHLs. To prepare the inocula for the motility, adhesion, and biofilm assays, the overnight culture medium was adjusted to a cell density of $1 \times 10^{7} \mathrm{CFU} / \mathrm{mL}$ based on counting colonies on the LB agar plate.

\section{Pyocyanin assay}

The production of pyocyanin pigment was measured using a quantitative chemical assay, as described by Essar et al. ${ }^{27}$ Briefly, bacteria from $1 \mathrm{~mL}$ of culture, as described earlier, were pelleted by centrifugation. The supernatant containing the pyocyanin was then extracted with $0.6 \mathrm{~mL}$ of chloroform, followed by extraction with $1 \mathrm{~mL}$ of $0.2 \mathrm{M} \mathrm{HCl}$ and measurement of the solution's $\mathrm{OD}_{520}$.

\section{LasA protease assay}

Proteolytic activity was measured as described by Hentzer et al. ${ }^{28}$ First, $250 \mu \mathrm{L}$ of $2 \%$ azocasein (Sigma-Aldrich) in $50 \mathrm{mM}$ Tris- $\mathrm{HCl}$ and a $150 \mu \mathrm{L}$ sterile-filtered supernatant, as described earlier, were incubated for 4 hours at $4{ }^{\circ} \mathrm{C}$. The undigested substrate was precipitated with $1.2 \mathrm{~mL}$ of $10 \%$ trichloroacetic acid for 15 minutes, followed by a 10-minute centrifugation at $10,000 \mathrm{rpm}$, and then $1.4 \mathrm{~mL}$ of $1 \mathrm{M} \mathrm{NaOH}$ was added to the supernatant. The relative protease activity was measured as the $\mathrm{OD}_{440}$ of the supernatant.

\section{LasB elastase assay}

The elastolytic activity of the LB culture supernatants was measured using an elastin Congo red (ECR; Sigma-Aldrich) assay as a substrate following the procedure previously described by Ohman et al. ${ }^{29}$ In brief, a $100 \mu \mathrm{L}$ aliquot was added to $900 \mu \mathrm{L}$ of ECR buffer (100 mM Tris, $1 \mathrm{mM} \mathrm{CaCl}_{2}$, $\mathrm{pH} 7.5)$ containing $20 \mathrm{mg}$ of ECR. This mixture was then incubated with agitation for 3 hours at $37^{\circ} \mathrm{C}$. Insoluble ECR was removed by centrifugation, and the absorption of the supernatant was measured at $495 \mathrm{~nm}$.

\section{Rhamnolipid assay}

Rhamnolipids in cell-free supernatants of $P$. aeruginosa PAO1 cultures were directly accessed by the orcinol method, 
as previously described..$^{30}$ Briefly, a $500 \mu \mathrm{L}$ sample of the culture supernatant was extracted twice with $1.5 \mathrm{~mL}$ of diethyl ether. The ether fractions were pooled and evaporated to dryness, and $500 \mu \mathrm{L}$ of $\mathrm{H}_{2} \mathrm{O}$ was added. A total of $900 \mu \mathrm{L}$ of a solution containing $0.19 \%$ orcinol (in $53 \%$ [v/v] $\mathrm{H}_{2} \mathrm{SO}_{4}$ ) was added to $100 \mu \mathrm{L}$ of each sample. After being heated in a water bath at $80^{\circ} \mathrm{C}$ for 30 minutes, the samples were cooled for 15 minutes at room temperature and the $\mathrm{OD}_{421}$ was measured. The concentrations of rhamnolipids were determined by comparing the data with the results obtained for rhamnose standards between 0 and $300 \mu \mathrm{g} / \mathrm{mL}$.

\section{Motility assay}

The swimming, swarming, and twitching motilities of $P$. aeruginosa $\mathrm{PAO} 1$ were measured using previously described methods. ${ }^{31}$ For the swimming assay, $1 \times 10^{7} \mathrm{CFU} / \mathrm{mL}$ $P$. aeruginosa $\mathrm{PAO} 1$ bacteria were inoculated with a sterile toothpick into the center of a $5 \mathrm{~mm} \mathrm{LB}$ medium plate containing $0.2 \%$ casamino acids $(\mathrm{w} / \mathrm{v}), 0.3 \%(\mathrm{w} / \mathrm{v})$ Bacto agar, and $30 \mathrm{mM}$ glucose. The swimming zone was measured after a 24 -hour incubation at $37^{\circ} \mathrm{C}$. To monitor swarming motility, bacteria were placed with sterile toothpicks into the centers of swarm plates consisting of $0.4 \%$ (w/v) Bacto agar and LB supplemented with $0.5 \%(\mathrm{w} / \mathrm{v})$ casamino acids and $0.5 \%(\mathrm{w} / \mathrm{v})$ glucose. For the twitching assay, bacteria were inoculated on the bottom of a petri dish by stabbing a toothpick through a thin $(2 \mathrm{~mm})$ layer of LB medium supplemented with $0.2 \%$ casamino acids, $30 \mathrm{mM}$ glucose, and $1.5 \%$ Bacto agar. After incubation for 24 hours at $37^{\circ} \mathrm{C}$, the agar was gently removed and the petri dish air-dried. A $1 \%$ crystal violet solution was used to stain the petri dish for 10 minutes. The petri dish was rinsed, and the crystal violet-stained twitching pattern was evaluated. The QS mutant $\Delta l a s I-\Delta r h l I$ was assayed as a negative control using the same procedure.

\section{Adhesion and biofilm-formation assay}

The adhesion of $P$. aeruginosa PAO1 to an abiotic surface was examined using previously described protocols. ${ }^{32,33}$ Experiments were conducted in 96-well flat-bottomed polystyrene microtiter plates (Corning Incorporated, Corning, NY, USA). P. aeruginosa PAO1 $\left(1 \times 10^{7} \mathrm{CFU} / \mathrm{mL}\right)$ was grown in the absence or presence of appropriate concentrations of baicalein $(32-128 \mu \mathrm{g} / \mathrm{mL})$ at $37^{\circ} \mathrm{C}$ under static conditions, and unadhered bacteria were rinsed off at 2-hour intervals beginning at 0 hours. The remaining bacterial biomass was stained with $1 \%$ crystal violet for 5 minutes and gently rinsed three times with sterile PBS. The crystal violet-stained wells were then dissolved in ethanol, and the bacterial concentration was measured spectrophotometrically using a wavelength of $595 \mathrm{~nm}$.

The effects of baicalein on preexisting premature (1-day-old) and mature (5-day-old) P. aeruginosa PAO1 biofilms were also assessed in flat-bottom 24-well polystyrene cell-culture plates (Corning) for quantification by crystal violet assay, or for scanning electron microscopy (SEM) studies on $13 \mathrm{~mm}$-diameter glass coverslips (Nest Biotechnology Co Ltd, Wuxi, People's Republic of China) placed into 24-well cell-culture plates. Each aliquot of $1 \times 10^{7} \mathrm{CFU} / \mathrm{mL}$ $P$. aeruginosa $\mathrm{PAO} 1$ was grown for 24 hours or 5 days, and unattached bacteria were gently rinsed off with PBS. Baicalein at appropriate concentrations $(32-128 \mu \mathrm{g} / \mathrm{mL})$ was added to the wells and incubated with the preexisting biofilm at $37^{\circ} \mathrm{C}$ for 24 hours without shaking. The biofilm biomass was quantified by staining with crystal violet following the method described earlier. For SEM observation, the biofilm-coated coverslips were fixed with $2.5 \%$ glutaraldehyde and dehydrated using a graded ethanol series $(50 \%, 70 \%, 80 \%, 90 \%$, and $100 \%)$. Finally, the samples were placed in a vacuum desiccator and coated by gold sputtering, followed by observation using SEM (SU8020; Hitachi Ltd, Tokyo, Japan) at $20 \mathrm{kV}$.

\section{Alginate assay}

Alginate estimation was performed according to the method of Franklin and Ohman. ${ }^{34}$ Alginates were collected from 1-day and 5-day supernatants of $P$. aeruginosa PAO1 biofilms incubated with or without different concentrations of baicalein, as described earlier. The collected alginates were precipitated using an equal volume of $2 \%(\mathrm{w} / \mathrm{v})$ cetylpyridinium chloride followed by centrifugation at 10,000 rpm for 20 minutes. Each pellet was suspended and precipitated twice with $5 \mathrm{~mL}$ of precooled isopropanol for purification. The concentration of alginate in solution was determined using the carbazole method of Knutson and Jeanes ${ }^{35}$ and using spectrophotometric measurements at $500 \mathrm{~nm}$ with an alginate reference substance (Sigma-Aldrich) as a standard.

\section{RNA extraction and real-time PCR assay}

To determine the expression levels of QS genes (lasI, lasR, $r h l I$, and $r h l R$ ), total RNA was extracted from $P$. aeruginosa PAO1 cells using an RNA MiniPrep kit (Corning). Residual DNA was removed by DNase I treatment (Thermo Fisher Scientific) following the manufacturer's recommendations. First-strand complementary DNA (cDNA) was synthesized using the RevertAid First Strand cDNA synthesis kit (Thermo Fisher Scientific) according to the manufacturer's instructions. Quantitative real-time polymerase chain reaction (PCR) was performed using SYBR Green II (Takara 
Holdings, Kyoto, Japan) on an ABI 7500 real-time PCR system (Thermo Fisher Scientific) with the specific primers listed in Table S1. To calculate the relative expression levels of the target genes, the expression level of the 16S ribosomal RNA gene was used as an internal control and calculated using the $2^{-\Delta \Delta \mathrm{Ct}}$ method described by Sarabhai et al. ${ }^{36}$ The results were obtained from three independent experiments.

\section{Acyl-homoserine lactone assay}

AHLs were extracted from the culture supernatants of $P$. aeruginosa PAO1 and its mutants deficient in las $R-r h l R$, and were incubated in the absence or presence of appropriate concentrations of baicalein, as described earlier. The extraction was performed with acidified ethyl acetate, and the resulting extracts were dried under nitrogen and quantified by HPLC electrospray mass spectroscopy (Alliance 2695/Quattro microsystem; Waters Corporation, Milford, MA, USA) following methods adapted from Makemson et $a{ }^{37}$ The peak intensities for odDHL $(\mathrm{m} / \mathrm{z}=298)$, BHL $(\mathrm{m} / \mathrm{z}=172)$, and specific ions, including an ion corresponding to the lactone moiety $\left([\mathrm{M}+\mathrm{H}]^{+}, \mathrm{m} / \mathrm{z}=102\right)$ and an ion derived from the acyl-chain moiety $\left([\mathrm{M}+\mathrm{H}-101]^{+}, \mathrm{m} / \mathrm{z}=197\right.$ or 71$)$, were combined and converted to concentrations using a standard curve generated from the pure compounds (Figure $\mathrm{S} 1$ and Table S2). Background readings from samples extracted with alkaline ethyl acetate were subtracted from the readings of the acid-extracted bacterial cultures before conversion, because the lactone ring is broken by alkaline hydrolysis, making AHLs too polar to be fully extracted into ethyl acetate.

\section{Animals}

Adult male Wistar rats (weighing 250-300 g) were obtained from the Laboratory Animal Center of Guangxi University (Nanning, People's Republic of China), raised in Makrolon cages, given free access to tap water and food, and maintained under a controlled environment $\left(22^{\circ} \mathrm{C} \pm 2^{\circ} \mathrm{C}\right.$ temperature and $60 \% \pm 10 \%$ humidity) with a 12 -hour light-dark cycle. The protocol in this study was conducted in accordance with the Guide for the Care and Use of Laboratory Animals published by the US National Institutes of Health (NIH publication 85-23, revised 1996), and was approved by the Animal Care and Use Committee of Guangxi Medical University, People's Republic of China.

\section{Macrophage cell isolation, culture, and infection by $P$. aeruginosa PAOI}

Primary culture of macrophages isolated from spleens of male Wistar rats was conducted using a magnetic-activated cell-sorting assay. ${ }^{38}$ Briefly, male Wistar rats were killed by cervical dislocation, and their spleens were extracted under aseptic conditions. The fibrous capsule and connective tissue were removed as much as possible, and the splenic tissue was cut into small segments (approximately 1-2 $\mathrm{mm}^{2}$ ) and mechanically triturated in precooled Dulbecco's Modified Eagle's Medium (DMEM; Thermo Fisher Scientific) to obtain a cell suspension. After centrifugation at 1,500 rpm for 5 minutes, erythrocytes were scavenged by resuspending the cell precipitation in ACK buffer (Thermo Fisher Scientific) for 30 minutes on ice. Mononuclear cells were washed and resuspended in MACS buffer (PBS containing 0.5\% [w/v] bovine serum albumin, $2 \mathrm{mM}$ ethylenediaminetetraacetic acid, $\mathrm{pH}$ 7.2). Then, the cells were passed through a $70 \mu \mathrm{m}$ nylon mesh (BD, Franklin Lakes, NJ, USA) to obtain a monocellular suspension. To remove lymphocytes, CD161.1-biotin (1:200), CD45RA-biotin (1:200), and CD3-biotin (1:200) antibodies (Abcam PLC, Cambridge, UK) were added, followed by incubation in the dark at $4{ }^{\circ} \mathrm{C}$ for 30 minutes. The cells were washed and sedimented by centrifugation at 1,500 rpm for 5 minutes. After resuspension in MACS buffer, $25 \mu \mathrm{L}$ of antibiotin microbeads per $10^{7}$ total cells (Miltenyi Biotec, Bergisch Gladbach, Germany) was added, and the resulting suspension was incubated at $4^{\circ} \mathrm{C}$ for 30 minutes. The cells were washed, centrifuged, and resuspended in DMEM. NK1.1 ${ }^{+}, \mathrm{CD}_{4} \mathrm{RA}^{+}$, and CD3 ${ }^{+}$ cells were depleted using an LD column (Miltenyi Biotec). The macrophages in the effluent were centrifuged at 1,500 rpm for 5 minutes, resuspended in DMEM supplemented with 10\% fetal bovine serum (Thermo Fisher Scientific) and antibiotics $(100 \mathrm{U} / \mathrm{mL}$ penicillin and $100 \mu \mathrm{g} / \mathrm{mL}$ streptomycin), and then further cultured at $37^{\circ} \mathrm{C}$ in a humidified incubator under $5 \% \mathrm{CO}_{2}$.

To establish a macrophage infection model, the purified macrophage suspensions $\left(1 \times 10^{6}\right.$ cells $/ \mathrm{mL}$ DMEM $)$ were seeded into six-well plates (Corning). Mid-log-phase $P$. aeruginosa $\mathrm{PAO} 1$ inocula were centrifuged, the cell pellet was washed three times with sterile PBS without $\mathrm{Ca}^{2+}$ or $\mathrm{Mg}^{2+}$ and resuspended in DMEM, and the colonies on an LB agar plate were counted prior to incubation. Macrophages were cocultured with $P$. aeruginosa PAO1 by adding the DMEM-adjusted bacterial suspension to the wells to achieve a multiplicity of infection (MOI) of $5 .^{39}$

\section{Enzyme-linked immunosorbent assay}

To quantify changes in the levels of cytokines, macrophages were cocultured with $P$. aeruginosa PAO1 (MOI =5) in the presence or absence of baicalein $(128 \mu \mathrm{g} / \mathrm{mL})$ for 4 hours at $37^{\circ} \mathrm{C}$. Macrophage-only cultures served as the 
untreated control, and macrophages incubated with baicalein $(128 \mu \mathrm{g} / \mathrm{mL})$ without PAO1 suspension served as the treatment control. After centrifugation at $4^{\circ} \mathrm{C}$ and $10,000 \mathrm{rpm}$ for 5 minutes, aliquots of the remaining supernatants were pipetted, and cytokine levels were quantified using commercial rat IL-1 $\beta$, IL-6, IL-8 and TNF $\alpha$ enzyme-linked immunosorbent assay (ELISA) kits (Thermo Fisher Scientific) following the manufacturer's instructions. Absorbance was measured at $450 \mathrm{~nm}$, and concentrations were calculated from the linear portion of the standard curves. The samples were assayed in duplicate, and each experiment was repeated at least three times.

\section{Preparation of total, cytoplasmic, and nuclear protein extracts}

The macrophages described in the ELISA section were centrifuged and washed twice with precooled PBS. Cytoplasmic and nuclear protein extracts were prepared using a Nuclear and Cytoplasmic Protein Extraction Kit (Sangon Biotech Co Ltd, Shanghai, People's Republic of China) according to the protocol described in the manufacturer's instructions. The protein concentrations in each fraction were estimated using a Bicinchoninic Acid Protein Assay Kit (Takara) following the manufacturer's instructions, with bovine serum albumin used as a standard.

\section{Western blot analysis}

Each fraction of proteins was prepared in Laemmli sample buffer (Sigma-Aldrich) and heated in boiling water for 5 minutes. All proteins were separated by sodium dodecyl sulfate polyacrylamide gel electrophoresis, electroblotted onto polyvinylidene difluoride membranes (Pall Corporation, Port Washington, NY, USA), and blocked (5\% nonfat milk in Tris-buffered saline with $0.1 \%$ Tween 20 ) for 60 minutes at room temperature. The membranes blotted with total proteins were then exposed separately to rabbit monoclonal primary antibodies against $\beta$-actin $(1: 1,000$, cell signaling technology [CST]), p38 (1:1,000, CST), phospho-p38 (1:1,000, CST), ERK1/2 (1:1,000, CST), phospho-ERK1/2 (1:1,000, CST), JNK (1:1,000, CST), phospho-JNK (1:1,000, CST); phospho-IкB $\alpha(1: 1,000$, CST), and $\operatorname{I\kappa B} \alpha(1: 1,000$, CST). The membranes blotted with cytoplasmic proteins were exposed to rabbit monoclonal primary antibodies against NFאB (p65) (1:500, CST) and $\beta$-actin $(1: 1,000$, CST), whereas the membranes blotted with nuclear proteins were exposed to rabbit monoclonal primary antibodies against NFאB (p65) (1:500, CST) and lamin B (1:500, Abcam). IRDye 800CW goat anti-rabbit IgG (H+L) (Li-Cor Biosciences, Lincoln, NE, USA) was used as the secondary antibody. The signal intensities of the bands were analyzed using the Odyssey infrared imaging system (Li-Cor).

\section{Measurement of the NFKB (p65) subunit in macrophage nuclear extracts}

The nuclear protein fraction from each experimental group of macrophages was used for the NFKB (p65) DNA-binding assay. The NFאB DNA-binding activity was assessed using a commercially available TransAM NFkB (p65) transcriptionfactor assay kit (Active Motif, Carlsbad, CA, USA) according to the protocols recommended by the manufacturer.

\section{Statistical analyses}

All experiments were repeated at least three times in duplicate to validate reproducibility. The graphs were constructed using GraphPad Prism software (version 5.0; GraphPad Software, Inc., La Jolla, CA, USA). All values are presented as mean \pm standard error. One-way analysis of variance was performed using SPSS 18.0 software (SPSS, Inc., Chicago, IL, USA) to compare differences between groups, followed by Dunn's post hoc test. $P$-values less than 0.05 were considered significant.

\section{Results}

\section{Minimum inhibitory concentration and} growth curves of $P$. aeruginosa PAOI in the presence of baicalein

The MIC of baicalein against $P$. aeruginosa $\mathrm{PAO} 1$ was greater than $1,024 \mu \mathrm{g} / \mathrm{mL}$. As shown in Figure 2, at concentrations of at least $256 \mu \mathrm{g} / \mathrm{mL}$, cell density and growth were significantly

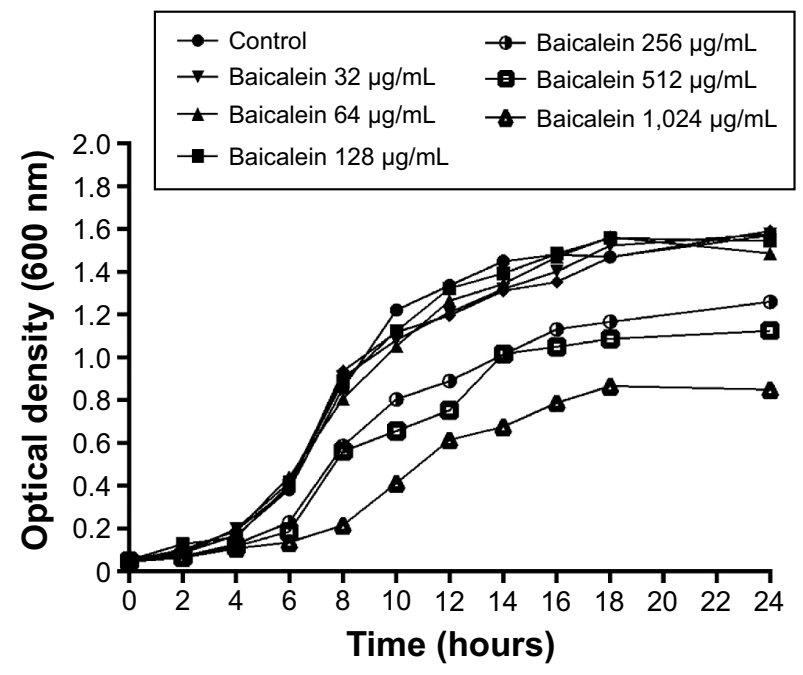

Figure 2 Growth curves of Pseudomonas aeruginosa PAOI incubated with different concentrations of baicalein.

Notes: Growth curves of P. aeruginosa PAOI in Luria-Bertani broth media with different concentrations of baicalein $(32-1,024 \mu \mathrm{g} / \mathrm{mL})$ or without baicalein (control) at $37^{\circ} \mathrm{C}$ for 24 hours. Experiments were performed in triplicate. The mean values are shown. With the exception of the groups treated with $\geq 256 \mu \mathrm{g} / \mathrm{mL}$ baicalein, there were no significant differences among the groups; therefore, the error bars are omitted for clarity. 
inhibited $(P<0.05)$ compared with the control group and with all lower baicalein concentrations. However, 32, 64, or $128 \mu \mathrm{g} / \mathrm{mL}$ baicalein had no significant impact on growth $(P>0.05)$. Therefore, $128 \mu \mathrm{g} / \mathrm{mL}$ was designated as the highest sub-MIC of baicalein. Because the MIC of baicalein is not a feasible concentration for use as an antibiotic, we focused on sub-MIC amounts of baicalein in the following experiments.

\section{Sub-MIC levels of baicalein suppress the production of extracellular virulence factors controlled by QS}

The presence of sub-MIC levels of baicalein attenuated the production of extracellular virulence factors by $P$. aeruginosa PAO1 (Figure 3). Our data showed that the expression levels of pyocyanin $(69.87 \%$ decrease), LasA protease $(74.56 \%$ decrease), LasB elastase ( $94.17 \%$ decrease), and rhamnolipid (74.15\% decrease) were significantly suppressed by $128 \mu \mathrm{g} / \mathrm{mL}$ baicalein. Moreover, the inhibitory effect of baicalein on the latter three QS-controlled virulence factors was dose-dependent, whereas the production of pyocyanin was significantly inhibited by $64 \mu \mathrm{g} / \mathrm{mL}$ baicalein. To investigate further whether the inhibitory effect of baicalein could be reversed by supplementation with exogenous AHLs, either synthetic odDHL (0.13 $\mu \mathrm{M})$ or BHL (5.2 $\mu \mathrm{M}$; SigmaAldrich) was added to the cultures in the early exponential phase; these doses are the maximal autoinducer concentrations for $P$. aeruginosa PAO1. ${ }^{40}$ Interestingly, our results revealed that the addition of odDHL relieved the inhibitory effect of baicalein on the secretion of all four extracellular virulence factors described earlier, whereas BHL significantly antagonized the inhibitory effects of baicalein on the production of pyocyanin and rhamnolipids.

\section{Effects of baicalein on the motility of P. aeruginosa PAOI}

$P$. aeruginosa displays three major forms of motility: 1) flagellum-mediated swimming in aqueous environments, 2) swarming on semisolid or viscous surfaces, and 3) total-factor

B
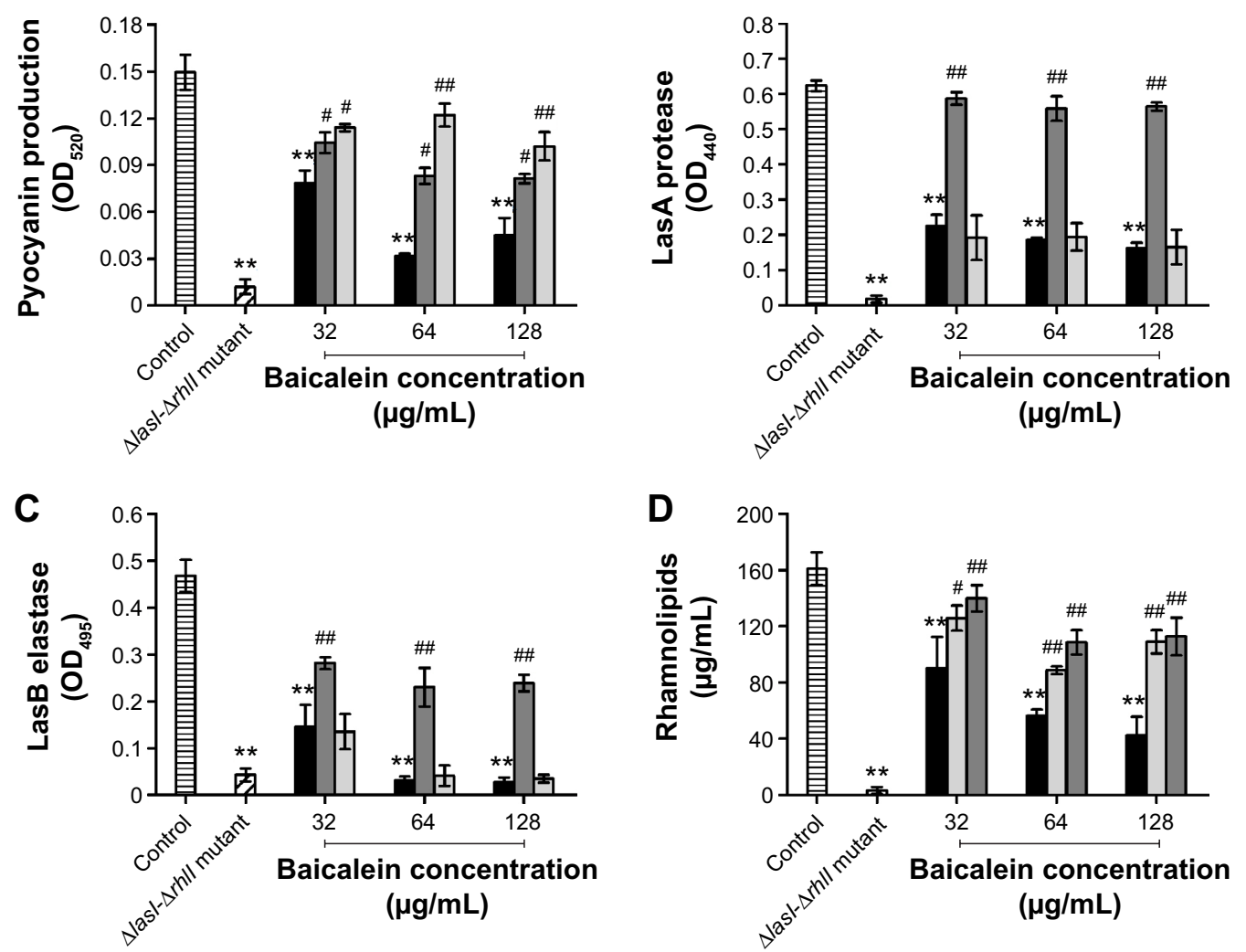

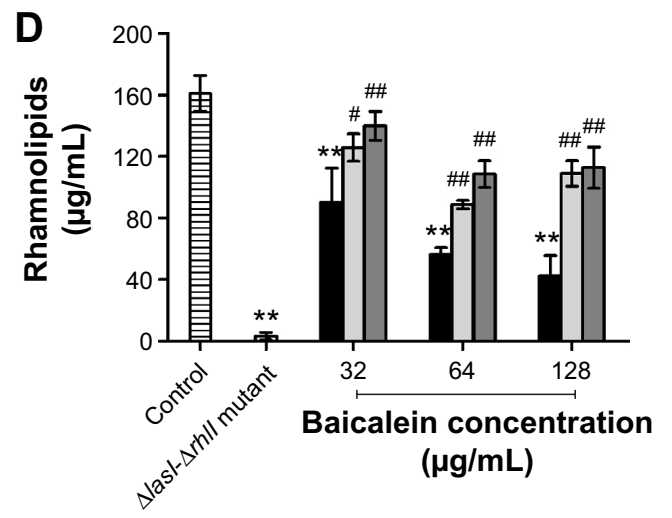

Е Control घ

Baicalein alone

Baicalein + odDHL

Baicalein $+\mathrm{BHL}$

Figure 3 Inhibitory effects of sub-minimum inhibitory concentration levels of baicalein on the production of quorum sensing-regulated extracellular virulence factors in Pseudomonas aeruginosa culture supernatants.

Notes: (A) Pyocyanin contents. (B) LasA protease activities. (C) LasB elastase activities. (D) Rhamnolipid contents. $* * P<0.01$ compared with the drug-free control group; ${ }^{\#} P<0.05$ and ${ }^{\# P}<0.01$ compared with the same concentration of baicalein alone. The bars indicate the standard error for triplicate sets of experiments.

Abbreviations: OD, optical density; odDHL, $\mathrm{N}$-(3-oxododecanoyl)-L-homoserine lactone; BHL, N-butanoyl-L-homoserine lactone. 
productivity mediated twitching on solid surfaces..$^{41}$ Notably, the mean swarming and twitching diameters in the presence of sub-MIC $(128 \mu \mathrm{g} / \mathrm{mL})$ levels of baicalein were significantly less than those of drug-free controls. Moreover, baicalein significantly reduced the capacity for these two types of motility in a manner similar to the $\Delta l a s I-\Delta r h l I$ mutant. Unexpectedly, the results showed that baicalein did not inhibit the swimming ability of $P$. aeruginosa PAO1; if anything, it exerted a weak (and not significant) stimulatory effect (Figure 4).

\section{Sub-MIC levels of baicalein reduce adhesion, detach the biofilm, and inhibit the alginate production of $P$. aeruginosa PAOI}

The adhesion of $P$. aeruginosa cells to an abiotic or biotic surface is the initial step following the microcolony and three-dimensional community structure of biofilm formation and maturation. ${ }^{42}$ Our results (Figure 5) revealed that the $\Delta$ lasI- $\Delta$ rhlI mutant strain exhibited a weaker adhesive ability

A

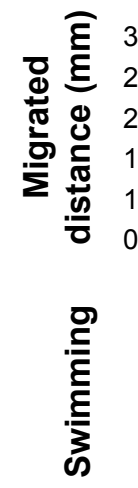

than the wild-type PAO1 strain throughout the 10 hours of observation. Notably, compared with the drug-free control, the number of $P$. aeruginosa PAO1 cells adhering to the surface of the 96-well plate was reduced by sub-MIC amounts of baicalein $(32-128 \mu \mathrm{g} / \mathrm{mL})$ in a concentration-dependent manner. Specifically, $128 \mu \mathrm{g} / \mathrm{mL}$ baicalein clearly inhibited the adhesion of $P$. aeruginos PAO1 to the abiotic surface at 2 hours, whereas $32-64 \mu \mathrm{g} / \mathrm{mL}$ began to exhibit effects at 4 hours. Crystal violet staining and quantification of the biofilm biomass remaining after treatment indicated that sub-MIC levels of baicalein detached both the 1-day-old (early phase) and 5-day-old (mature phase) biofilms in a concentration-dependent manner (Figure 6). The highest sub-MIC amount of baicalein $(128 \mu \mathrm{g} / \mathrm{mL})$ showed the strongest effect, reducing the 1-day and 5-day biomass of the wild-type PAO1 strain by approximately $35.7 \%$ and $53.0 \%$, respectively. Subsequently, alterations in the structure of biofilms incubated in the presence or absence of baicalein $(128 \mu \mathrm{g} / \mathrm{mL}$ ) were observed using SEM (Figure 7). In the

B

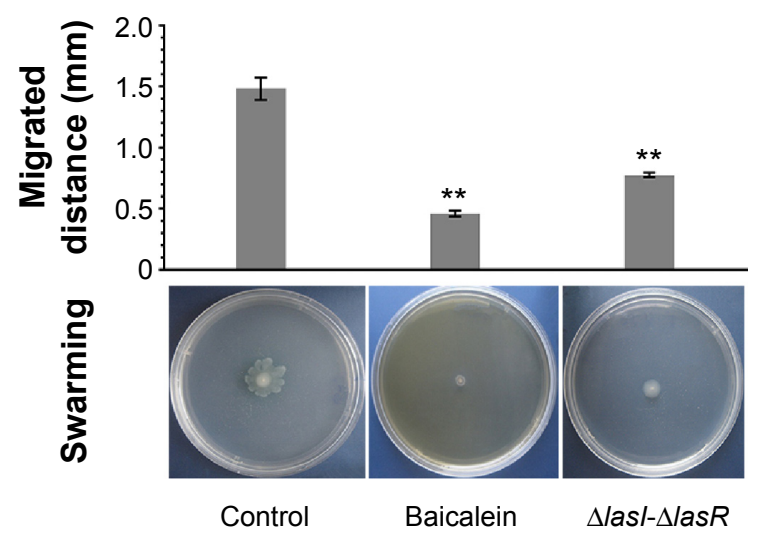

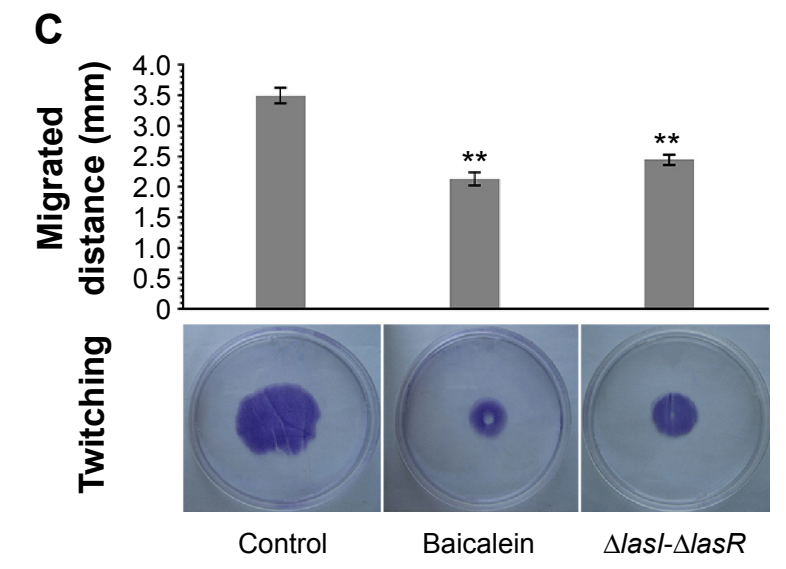

Figure 4 The effects of baicalein on the motility of Pseudomonas aeruginosa.

Notes: Motility assays were conducted on plates containing different concentrations of agar in the absence of baicalein (drug-free control) or with $128 \mu \mathrm{g} / \mathrm{mL}$ baicalein. Diameters of (A) swimming, (B) swarming, and (C) twitching motilities were measured using a caliper. The data represent the average value of three independent experiments in duplicate. Values are mean \pm standard error. $* * P<0.01$ compared with the drug-free control group. 


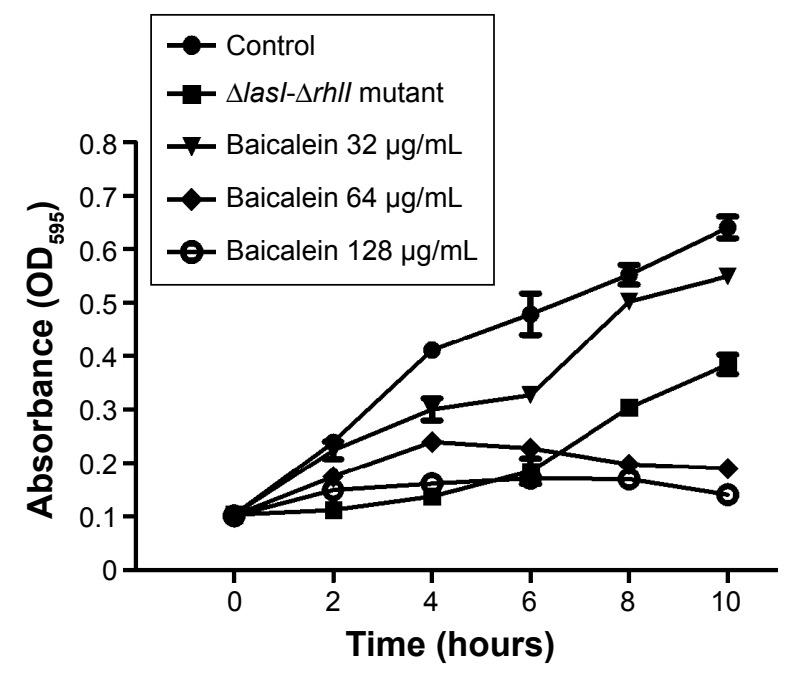

Figure 5 Effects of different concentrations of baicalein on the adhesion of Pseudomonas aeruginosa to an abiotic surface.

Notes: Cell adhesion was indirectly evaluated using a crystal violet assay at $\mathrm{OD}_{595}$ at 2-hour intervals from the beginning of the treatment until the tenth hour. The value of each group at each time point is presented as the mean \pm standard error. The data were obtained from triplicate sets of experiments.

Abbreviation: OD, optical density.

drug-free condition, a three-dimensional structure was clearly visible in the biofilm formed by PAO1, and the extracellular polysaccharide (EPS) matrix was denser and more compact than that of $\Delta$ lasI- $\Delta r h l I$ after growing for either 1 or 5 days. By contrast, the EPS matrix was obviously reduced, and PAO1 cells from the detached biofilm mass were dispersed into a scattered state after exposure to baicalein. Furthermore, we investigated alginate production in the biofilm supernatants; the results showed a trend that was synchronous with that of the biofilm quantification (Figure 8).

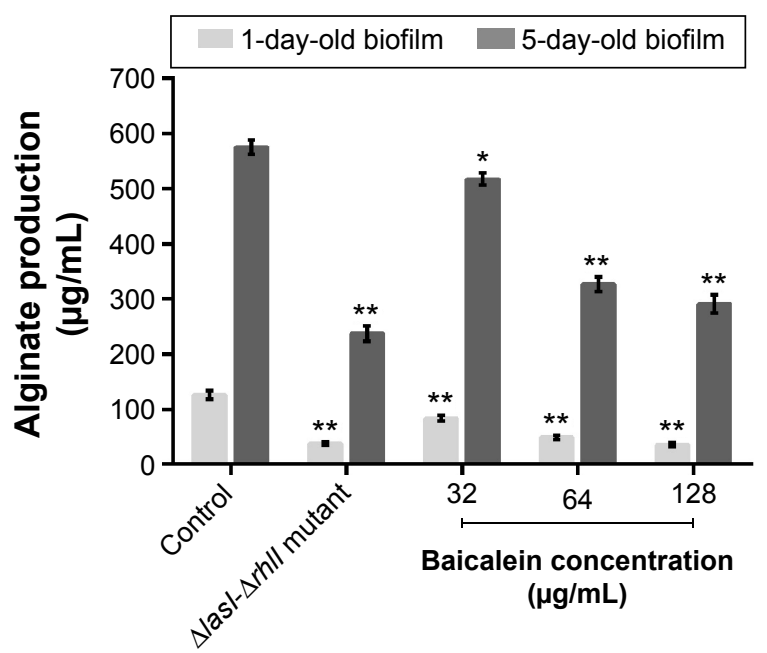

Figure 6 Biofilm quantification by crystal violet assay.

Notes: The data represent the average values of three independent experiments. Values are mean \pm standard error. $* P<0.05$ and $* * P<0.01$ compared with the drugfree control group.

\section{Mechanism of the anti-QS activity of baicalein}

The four target QS-regulated genes of $P$. aeruginos a control the expression of numerous QS-related virulence factors and the production of AHL, and they play important roles in the development of biofilms. Our real-time PCR data showed that the transcription levels of lasI, lasR, rhlI, and rhlR were downregulated by $69.6 \%, 80.8 \%, 64.6 \%$, and $72.5 \%$, respectively, after incubation with $128 \mu \mathrm{g} / \mathrm{mL}$ of baicalein (Figure 9), indicating that baicalein might globally inhibit the QS system of $P$. aeruginosa. In addition, the inhibitory effects of baicalein on the expression of the lasI, rhlI, and rhlR genes occurred in a concentration-dependent manner. The production of AHLs, as analyzed by HPLC mass spectroscopy (Figure 10), supported the gene-transcription results. Reductions in odDHL by $63.2 \%$ and $58.7 \%$ in BHL were detected after exposure to sub-MIC levels of baicalein. These reductions in both AHLs and QS-related genes were consistent with the reductions in virulence factors directly controlled by odDHL (protease and elastase) and BHL (pyocyanin and rhamnolipids) in P. aeruginosa PAO1.

\section{Baicalein attenuates the inflammatory response in P. aeruginosa PAOI-infected macrophages}

To address the potential therapeutic efficacy of baicalein, its anti-inflammatory activities and mode of action were examined in primary splenic cells of rats stimulated with $P$. aeruginosa PAO1. Macrophages play a critical role in the response to bacterial pathogen infection. As the first immune cells to encounter $P$. aeruginosa, macrophages are activated largely based on the well-known recognition of pathogen-associated molecular patterns. ${ }^{43,44}$ The initial detection of the pathogen triggers not only phagocytosis but also a series of changes in gene expression and the production of inflammation-related cytokines, such as IL-1 $\beta$, IL-6, IL-10, and TNF $\alpha$, resulting in the recruitment and activation of inflammatory cells in response to $P$. aeruginosa infection. ${ }^{45}$ These cytokines are the main signaling mediators released by monocytes or macrophages. Our initial experiment confirmed that the most substantial secretions of IL-1 $\beta$, IL-6, IL-10, and TNF $\alpha$ were observed after rat primary macrophages were cocultured with $P$. aeruginosa $\mathrm{PAO} 1$ at $\mathrm{MOI}=5$ for 4 hours, which is consistent with the findings of Ortega-González et al ${ }^{38}$ Moreover, the highest sub-MIC level of baicalein $(128 \mu \mathrm{g} / \mathrm{mL})$ did not inhibit the growth of macrophages evaluated by the 3-(4,5-dimethylthiazol-2-yl)-2,5-diphenyltetrazolium bromide (MTT) assay in our preliminary experiment (Figure S2). 


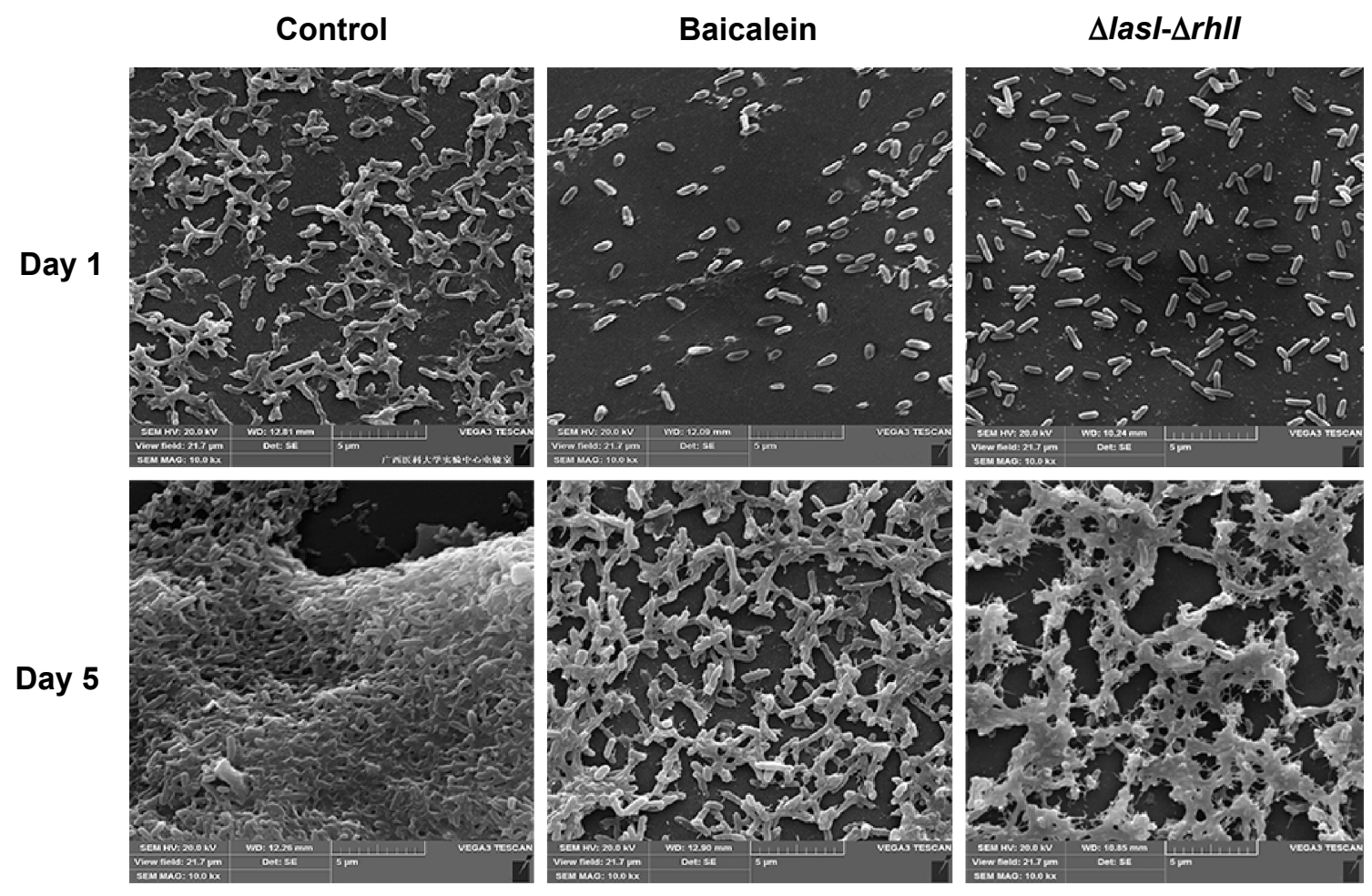

Figure 7 Scanning electron microscopy images of biofilms formed by Pseudomonas aeruginosa PAOI (I00,000 $\times$ magnification).

Notes: The I-day-old (early phase) and 5-day-old (mature phase) biofilms of $P$. aeruginosa PAOI in the absence and presence of baicalein (I $28 \mu \mathrm{g} / \mathrm{mL}$ ) and the biofilms formed by $\Delta$ lasl- $\Delta r h l l$ on the coverslip are shown.

Therefore, these experimental conditions were used to assess the effects of baicalein on the secretion of inflammatory cytokines and on inflammation-related signaling pathways.

As shown in Figure 11, the highest sub-MIC $(128 \mu \mathrm{g} / \mathrm{mL})$ level of baicalein showed no significant effect on cytokine secretion in the absence of $P$. aeruginosa, although slight increases or decreases were noted. As expected, PAO1

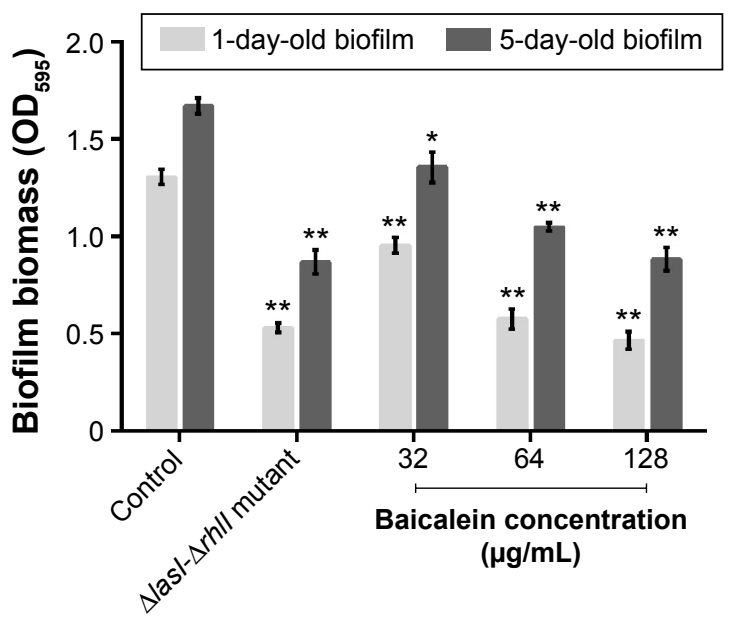

Figure 8 Effects of different concentrations of baicalein on alginate production by Pseudomonas aeruginosa.

Notes: Data represent mean \pm standard error of three independent experiments. $* P<0.05$ and $* * P<0.01$ compared with the drug-free control group. infection induced a significant increase $(P<0.01)$ in the secretion levels of all four target cytokines compared with the drug-free control group. The sub-MIC level of baicalein $(128 \mu \mathrm{g} / \mathrm{mL})$ significantly attenuated IL-1 $\beta$, IL-6, IL-8, and TNF $\alpha$ secretion compared with the PAO1-infected group in the absence of baicalein treatment.

\section{P. aeruginosa-induced activation of the MAPK pathway is downregulated by baicalein}

The MAPK-signaling pathway is involved in modulating inflammatory action responses to various stresses in exogenous pathogen infection, and consists of three major family members: the Perks, JNKs, and p38 MAPKs. ${ }^{46}$ We investigated the possible effect of baicalein on $P$. aeruginosa-mediated MAPK-pathway activation using Western blot analysis. As shown in Figure 12, there was a remarkable increase in the phosphorylation levels of p38 $(P<0.01)$, ERK1/2 $(P<0.01)$, and JNK $(P<0.01)$ in macrophages infected by $P$. aeruginosa PAO1. Interestingly, treatment with a sub-MIC level of baicalein $(128 \mu \mathrm{g} / \mathrm{mL})$ significantly $(P<0.05$ for the phospho-p38 ratio and $P<0.01$ for the phospho-ERK1/2 ratio and the phospho-JNK ratio) suppressed these changes compared with the $P$. aeruginosa PAO1-infected group without baicalein. 


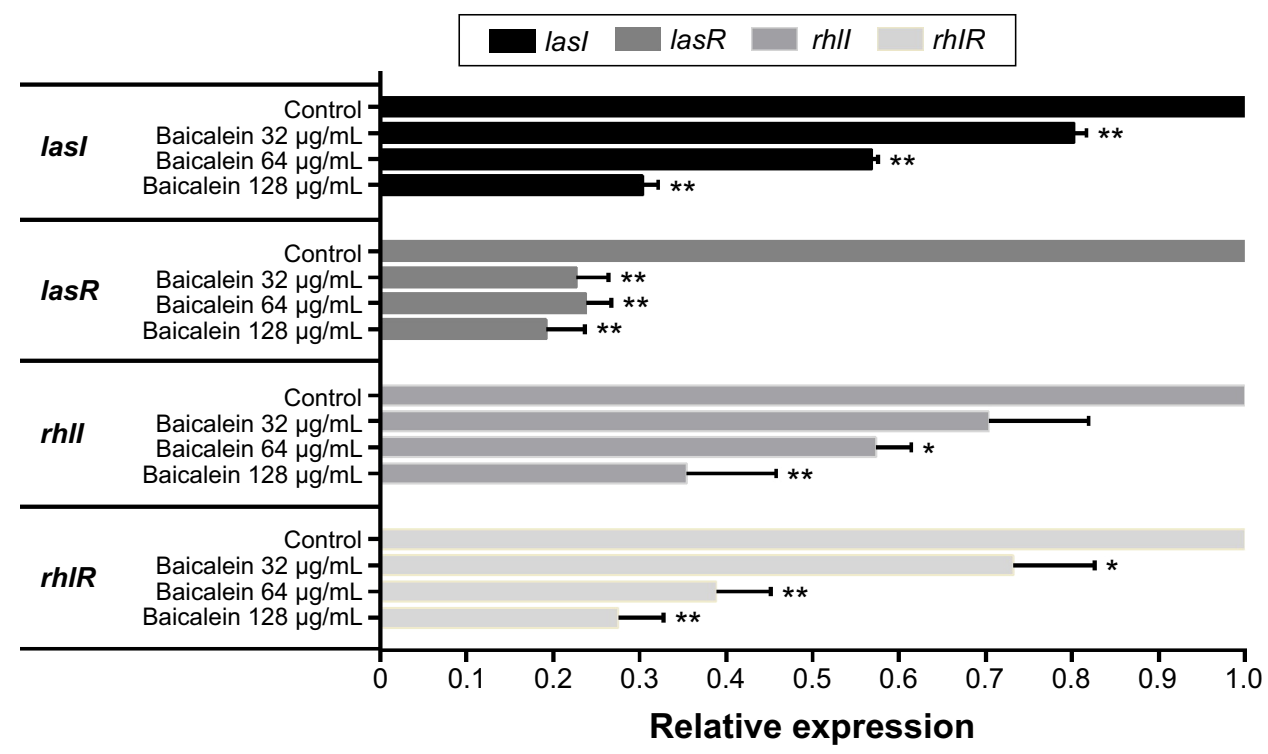

Figure 9 Relative expression levels of quorum sensing-regulated genes of Pseudomonas aeruginosa PAOI in the presence of sub-minimum inhibitory concentration levels of baicalein, as determined by real-time polymerase chain reaction.

Notes: The data represent the mean \pm standard error of three independent experiments. $* P<0.05$ and $* * P<0.01$ compared with the drug-free control group.

\section{Baicalein suppresses $P$. aeruginosa-induced

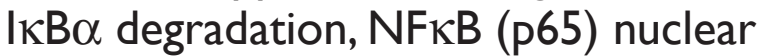 translocation, and NFKB binding}

One of the ways the NFKB-signaling pathway is activated by extracellular pathogen stimuli involves the rapid degradation of $I \kappa B \alpha$ due to I $\kappa \mathrm{B} \alpha$ phosphorylation at Ser32 by IKB $\alpha$ kinase, which corresponds to IKK in the "canonical" pathway. Ubiquitination then occurs, allowing NFkB (p65) to translocate to the nucleus, where NFKB (p65) binds to certain sites and triggers the transcriptional activation of inflammation-related genes. ${ }^{22}$ The activation of NFkB (p65) signaling also plays a key role in mediating inflammation through the induction of proinflammatory cytokines. ${ }^{22,38}$ As shown in Figure 13, there was a marked and significant $(P<0.05)$ increase in the phosphorylation of IKB $\alpha$ in the total protein from the $P$. aeruginosa $\mathrm{PAO}$-infected macrophages compared with the control group. In addition, there was a significant $(P<0.05)$ increase in NFKB (p65) accumulation in the nuclear fraction protein from the $P$. aeruginosa PAO1infected macrophages compared with the control group. The NFKB (p65) DNA-binding assay also confirmed the enhanced $(P<0.01)$ DNA-binding activity of the p65 subunit of NFKB
A

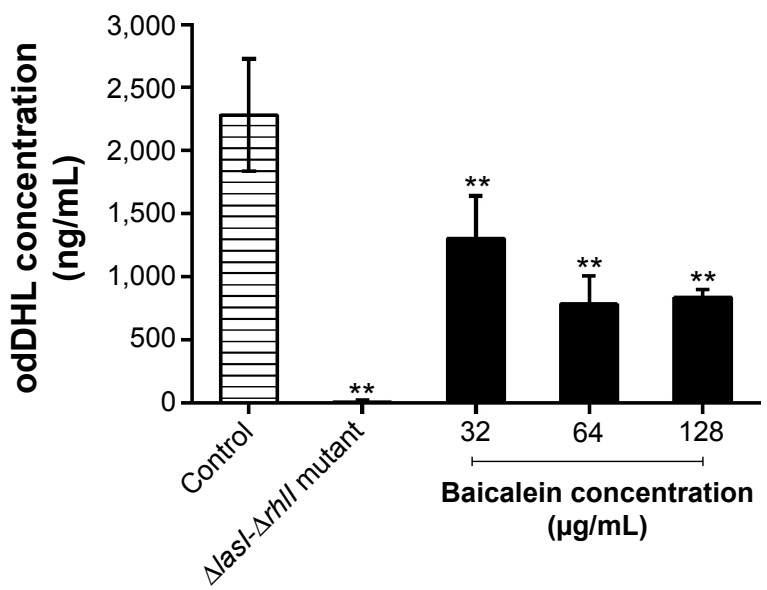

B

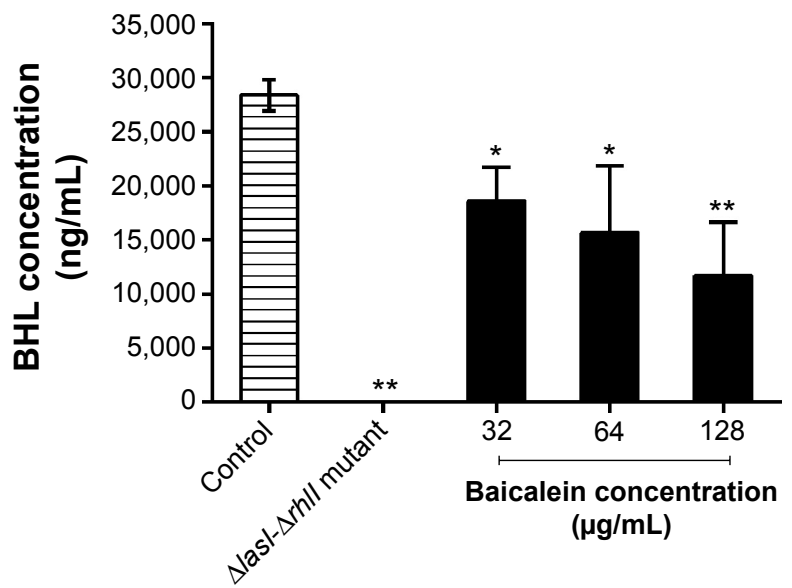

Figure 10 Baicalein interferes with quorum-sensing-signaling molecules $(\mathbf{A})$ odDHL and $(\mathbf{B}) \mathrm{BHL}$ in culture supernatants.

Notes: Data represent mean \pm standard error of three independent experiments. $* P<0.05$ and $* * P<0.01$ compared with the drug-free control group. Abbreviations: odDHL, $\mathrm{N}$-(3-oxododecanoyl)-L-homoserine lactone; BHL, $\mathrm{N}$-butanoyl-L-homoserine lactone. 
A
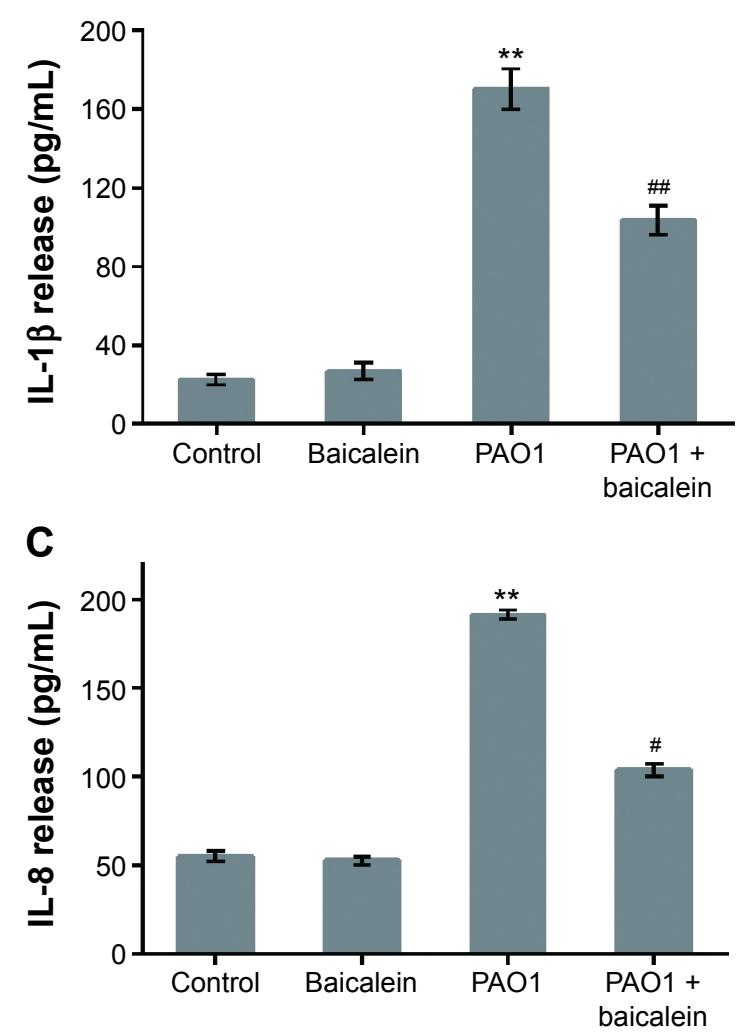

B
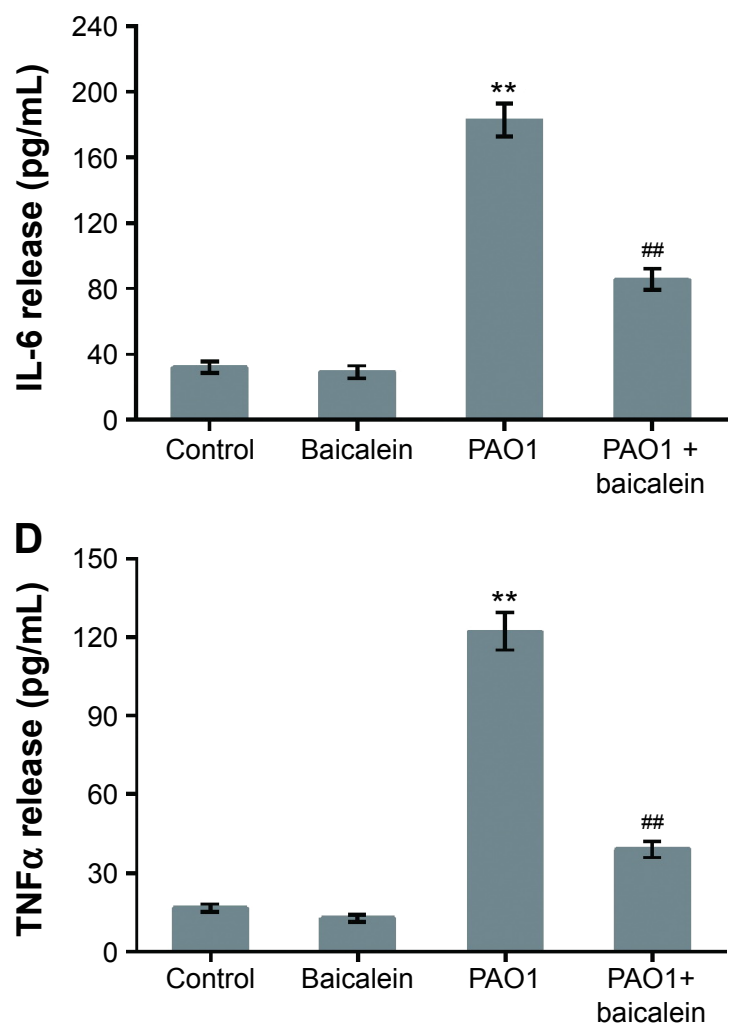

Figure I I Effects of baicalein on the production of inflammatory cytokines by Pseudomonas aeruginosa PAOI-infected macrophages.

Notes: Primary rat macrophages were cocultured with $P$. aeruginosa PAOI cells (multiplicity of infection $=5)$ for 4 hours in the absence or presence of baicalein $(I 28 \mu g / m L)$ prior to the detection of (A) IL-I $\beta$, (B) IL-6, (C) IL-8, and (D) TNF $\alpha$. Data represent mean \pm standard error of three independent experiments. $* * P<0.0$ I versus macrophages without bacteria or baicalein (control group); ${ }^{\#} P<0.05$ and ${ }^{\# P}<0.0$ I versus $P$. aeruginosa PAOI in the absence of baicalein.

in the nuclear fraction protein from the P. aeruginosa PAO1infected macrophages. Treatment with a sub-MIC level of baicalein $(128 \mu \mathrm{g} / \mathrm{mL})$ significantly prevented $P$. aeruginosainduced I $\mathrm{\kappa} B \alpha$ phosphorylation and the subsequent nuclear translocation and DNA-binding activity of NFKB (p65) compared with the $P$. aeruginosa PAO1-infected group without baicalein.

\section{Discussion}

With the extensive use and abuse of antibiotics, a serious problem of bacterial resistance has developed, resulting in a decreased curative effect of conventional antibiotics. ${ }^{47}$ However, plant-derived compounds have been used to treat infectious disease for centuries and have been proven effective, with low toxicity and low acquired resistance. ${ }^{16}$ Unlike most antibiotics, which function by directly killing bacterial cells, plant-derived compounds can act via various alternative mechanisms, eg, antivirulence strategies that disarm pathogens and generate less pressure for the evolution of resistance ${ }^{40}$ Therefore, the exploration of plant-derived compounds, especially monomer components with well-defined chemical structures, for application in treating microbial infection in the clinic is attractive.

$P$. aeruginosa is a common cause of severe nosocomial infections in lungs, wounds, and patients undergoing longterm ventilator-support therapy, and it is often intractable to treatment, due to the high prevalence of multidrug resistance. The pathogenesis of $P$. aeruginosa is closely associated with the production of a myriad of extracellular virulence factors, motility, and the formation of biofilm, all of which are regulated by QS systems in bacteria. ${ }^{5}$ Anti-QS strategies may provide a novel and promising means of fighting P. aeruginosa infection. Some QS inhibitors derived from plants or microbes, such as halogenated furanones, penicillic acid, patulin, and garlic extract, have been found to possess QS-inhibitor activity against $P$. aeruginosa in vitro or in vivo. ${ }^{48-50}$ However, these compounds have inherent drawbacks that make them unsuitable for the treatment of humans. Halogenated furanones are unstable and easily decomposed, penicillic acid and patulin are mycotoxins, and the active components in garlic are present in very low concentrations. Therefore, these compounds are unsuitable 

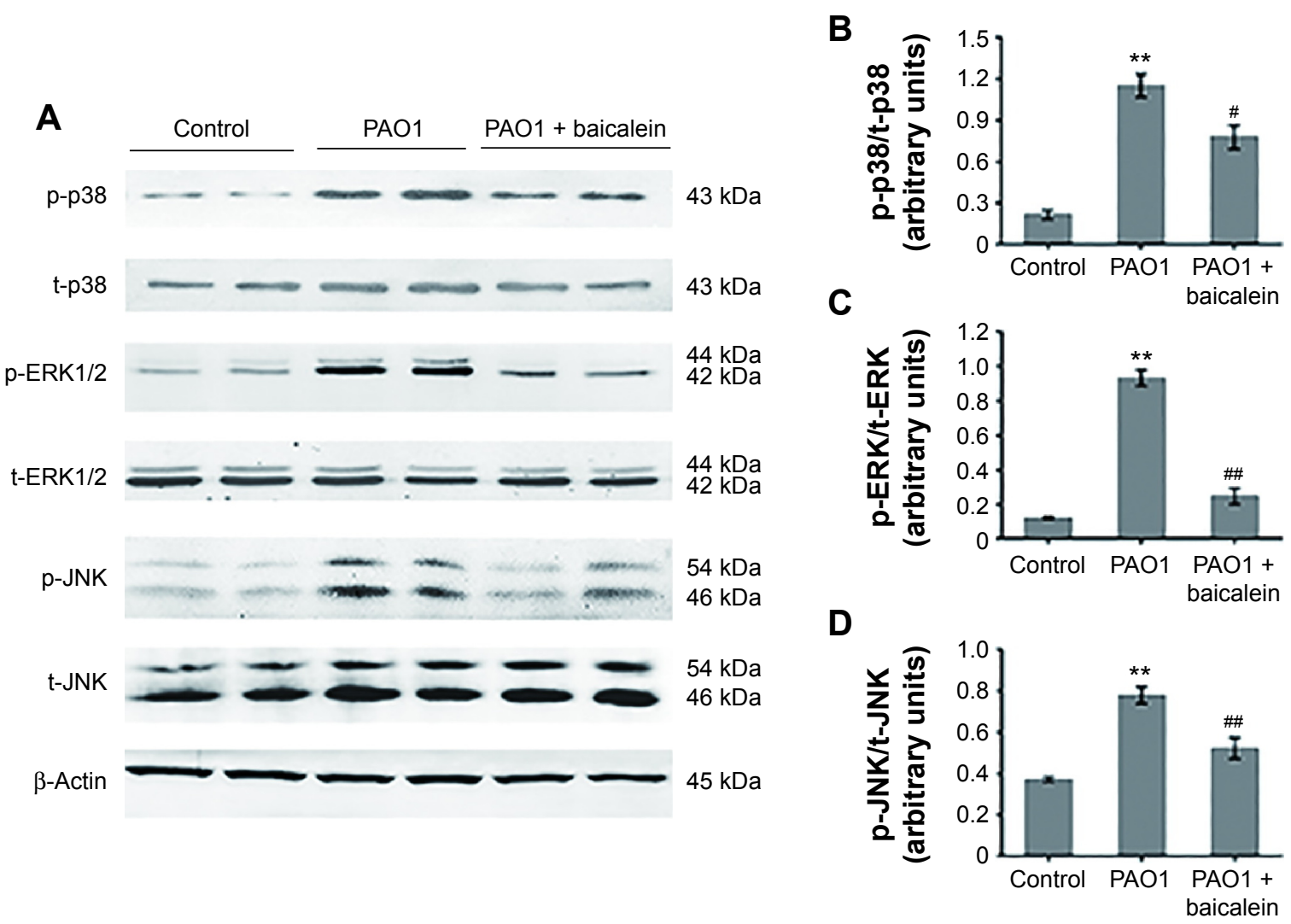

Figure 12 Effects of baicalein on the expression of MAPK pathway-related proteins in macrophages infected by Pseudomonas aeruginosa PAOI.

Notes: Immunoblot analyses showing the expression levels of p-p38, t-p38, p-ERKI/2, t-ERKI/2, p-JNK, and t-JNK (A). Duplicate immunoblot samples from one representative of three separate experiments are shown; $\beta$-actin was used as an internal control. Bar diagrams showing densitometric analysis of the relative expression of (B) p-p38/t-p38, (C) $\mathrm{p}$-ERK/t-ERK, and (D) $\mathrm{p}$-JNK/t-JNK, quantified using the Odyssey infrared system. Values are mean \pm standard error ( $\mathrm{n}=6$ ). $* * \mathrm{P}<0.0 \mathrm{I}$ versus macrophages without bacteria or baicalein (control group); ${ }^{\# P}<0.05$ and ${ }^{\# P} P 0.0$ I versus $P$. aeruginosa $P A O I$ in the absence of baicalein.

Abbreviations: p-, phospho-; t-, total.

for human use. In this study, we demonstrated the efficient effect of baicalein, one of the major flavonoid monomers of Scutellaria baicalensis, on the production of QS-regulated virulence factors, including the synthesis of signaling molecules and QS-regulated genes, independently of any effect on the growth of $P$. aeruginosa.

Secreted extracellular virulence factors, including pyocyanin, protease, elastase, and rhamnolipids, are considered indicators of the optimal operation of the QS regulon in $P$. aeruginosa. Moreover, they serve as key mediators of acute $P$. aeruginosa infection. ${ }^{36}$ Reductions in their levels indicate the anti-QS potential of the tested agent(s). Protease and elastase (regulated by the las system) play crucial roles in colonizing host tissues, ${ }^{51}$ whereas pyocyanin (regulated by the $r h l$ system) chelates the bound iron from transferrin for optimal virulence. ${ }^{52}$ Rhamnolipids (regulated by the $r h l$ system) constitute an important surfactant that assists in the surface motility of $P$. aeruginosa that is required for biofilm initiation..$^{53}$ The data from this study showed that baicalein reduced all of these factors to levels comparable to those of the $\Delta$ lasI- $\Delta$ rhlI mutant strain, indicating the effectiveness of this compound in attenuating $P$. aeruginosa virulence factors and its potential for treating acute $P$. aeruginosa infection. Interestingly, supplementation with exogenous synthetic AHLs relieved the inhibitory effect on virulence factors, indicating that the attenuation of $P$. aeruginos $a$ virulence by baicalein may be partially due to the decreased synthesis of AHLs, which are critical signaling molecules that activate the QS pathway and the production of various virulence factors. It has even been reported that Yunnan baiyao, a complex Chinese medicine widely used for healing wounds and relieving pain, exerts remarkable effects against QS-related extracellular virulence factors; ${ }^{40}$ however, the lack of verification of the effective constituent limits its practical use.

Biofilms can be considered highly organized bacterial communities encased in exopolysaccharides and attached to a surface. ${ }^{10,36,38}$ The formation of a biofilm occurs in three major stages: 1) reversible to irreversible adhesion; 2) EPSmatrix encasement of the microcolonies and the early stage of biofilm formation; and 3) biofilm maturation..$^{42}$ Once the 


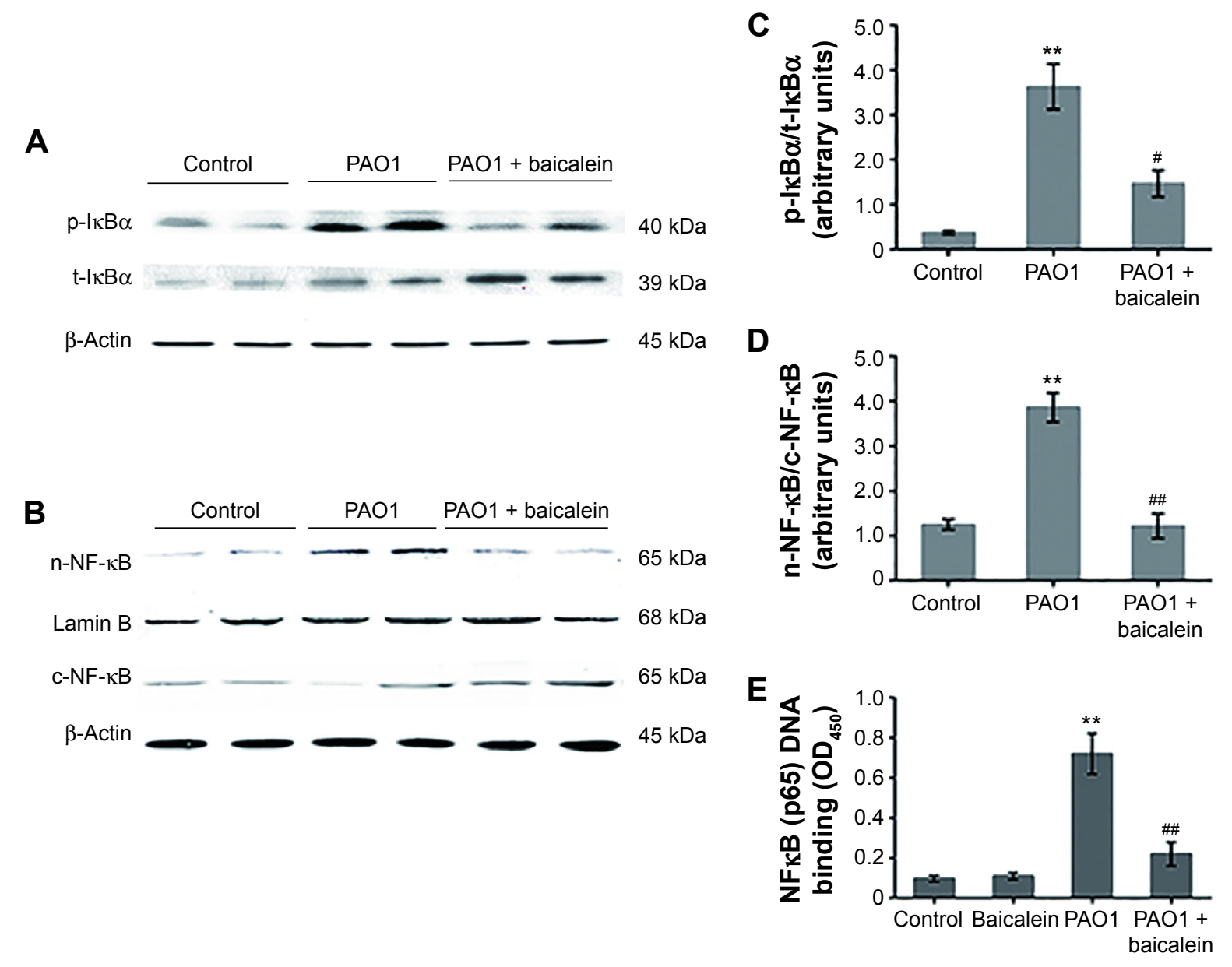

Figure 13 Effects of baicalein on the expression of NFKB-related proteins in macrophages infected by Pseudomonas aeruginosa PAOI.

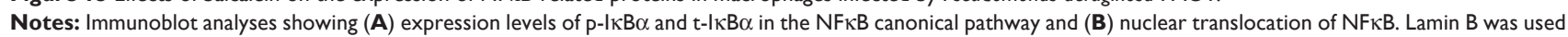
as the internal control for the nuclear fraction, and $\beta$-actin was used as the internal control for cytoplasmic and total protein fractions. Duplicate immunoblot samples

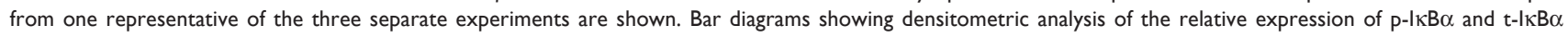
(C) and $n-N F-\kappa B / c-N F-\kappa B(D)$, quantified using the Odyssey infrared system. (E) DNA binding activity of p65 subunit of NFKB in the nuclear protein fractions. Values are mean \pm standard error $(n=6)$. $* * P<0.0$ I versus macrophages without bacteria or baicalein (control group); ${ }^{*} P<0.05$ and ${ }^{\#} P<0.0 \mathrm{I}$ versus $P$. aeruginosa $P A O \mathrm{I}$ in the absence of baicalein.

Abbreviations: p-, phospho-; t-, total; n-, nuclear; c-, cytoplasmic.

biofilm is established, the $P$. aeruginosa cells embedded in it are highly resistant to antibiotic treatment, which also contributes to recurrence, and thus biofilms are considered critical factors in chronic infection. ${ }^{54}$ Alginate is present as a constituent of the extracellular matrix of $P$. aeruginosa biofilms, and is an important virulence determinant regulated by QS. ${ }^{30,51}$ In this study, a sub-MIC level of baicalein inhibited the initial adhesion of planktonic cells to an abiotic surface to block the initial stage of biofilm formation. The quantification experiment showed that baicalein significantly reduced the biomass of static $P$. aeruginosa biofilms at both early and mature stages in a dose-dependent manner. Likewise, SEM revealed that baicalein treatment led to the detachment of the biofilm structure, indicating that baicalein may be used for the prevention and treatment of chronic infection caused by $P$. aeruginosa biofilms. The alteration of alginate production also correlated well with the change in biofilm biomass after sub-MIC baicalein treatment, indicating that this may be the mechanism by which baicalein led to the detachment of different ages of $P$. aeruginosa biofilm. This dispersing behavior of baicalein against sessile biofilm cells may also increase the planktonic bacterial cell count in the medium without EPS-matrix encasement, making the bacteria vulnerable to the actions of antibiotics.

Motility is crucial for colonization, the expression of full virulence, and biofilm formation; therefore, it plays an important role in the pathogenesis of $P$. aeruginosa. ${ }^{31,40,41}$ Although baicalein did not reduce the swimming ability of P. aeruginosa, it significantly affected swarming and twitching motilities, which suggests that baicalein can interfere with the functions of flagella and type IV pili. Because the motility of $P$. aeruginosa is regulated by the QS system, ${ }^{55}$ baicalein 
may inhibit motility by either interfering with QS or acting directly on type IV pili and flagella, thus partially contributing to the inhibition of adhesion, to biofilm dispersion, and to the reduced expression of other virulence factors.

The QS system can be inhibited in various ways. A virtual screening study conducted by Zeng et $\mathrm{al}^{25}$ reported that baicalein could promote the proteolysis of the QS signal receptor TraR protein, which may be the mechanism underlying this process. Here, we investigated the QS targets of baicalein by quantifying the transcription levels of QS genes and the translation levels of QS-signaling molecules. Our results revealed that the expression levels of all four QS genes (lasR, lasI, rhlR, and lasI) and the production of two QS-signaling molecules (odDHL and BHL) were significantly downregulated by sub-MIC amounts of baicalein in a dose-dependent manner. These findings indicate that baicalein may possess a global inhibitory effect on $P$. aeruginosa QS-signaling and QS-related virulence factors.

Microbial infections are dependent on an array of interactions between the pathogen and the host immune system. Therefore, an antimicrobial agent should not target only the former, because the latter may influence therapeutic prognosis. It has been reported that $P$. aeruginosa infection frequently aggravates patient symptoms, resulting in organ dysfunction, subsequent systemic inflammatory reaction syndrome, and increased morbidity and mortality., This association may be greatly attributable to the triggering of the inflammatory cascade when macrophages first encounter the $P$. aeruginosa bacteria, followed by the release of proinflammatory cytokines, including IL- $1 \beta$, IL-6, IL-8, and TNF $\alpha$, subsequently attracting a massive influx of neutrophils to the focus of infection and inducing the production of other cytokines, which in turn mediate other inflammatory immune responses and tissue damage. ${ }^{13,45,56}$ Proinflammatory cytokine production by the macrophage lineage orchestrates the immune response and predicts the outcome of infection. ${ }^{13}$ In this study, treatment with a sub-MIC level of baicalein significantly reduced the $P$. aeruginos $a$-induced production of proinflammatory cytokines (IL-1 $\beta$, IL-6, IL-8, and TNF $\alpha$ ) by macrophages independently of affecting cell viability, indicating that baicalein might represent a novel promising anti-inflammatory agent to prevent $P$. aeruginosa-mediated inflammatory impairment.

The host recognition of pathogens by molecular pattern receptors may result in two entirely different outcomes. ${ }^{57} \mathrm{An}$ appropriate response leads to the clearance of the microorganism, whereas an exaggerated inflammatory response may lead to illness, such as sepsis and shock. ${ }^{58}$ Previous work has reported that the $P$. aeruginosa-induced activation of macrophages is largely based on the recognition of pathogens by molecular pattern receptors, including Toll-like receptors (TLRs), such as TLR2 and TLR $4,{ }^{43}$ and the mannose receptor, ${ }^{44}$ which contribute equally to proinflammatory cytokine production in response to viable $P$. aeruginosa bacterial infection via activation of the MAPK- and NFKBsignaling pathways. ${ }^{17}$ Moreover, the mannose receptor serves as a compensator in host protection against $P$. aeruginosa and synergizes with TLR2 for maximum MAPK and NFKB activation and proinflammatory cytokine production. ${ }^{17}$ A previous study has indicated that baicalein can attenuate acute renal injury by inhibiting the inflammation response via downregulating the MAPK- and NFKB-signaling pathways. ${ }^{22}$ Moreover, baicalein treatment also markedly relieves the inflammatory damage to the mammary gland induced by lipopolysaccharides and suppresses proinflammatory cytokine production in mice with lipopolysaccharide-induced mastitis by blocking the expression of the TLR4-mediated NFKB- and MAPK-signaling pathways. ${ }^{59}$ The present study demonstrates that baicalein can attenuate these induced inflammatory responses by inactivating the p38-, ERK1/2-, JNK-, and NFKB (p65)-signaling pathways, which is consistent with the reduction in proinflammatory cytokines secreted by $P$. aeruginosa-infected macrophages. These results suggest that the inhibition of the MAPK- and NFKBsignaling pathways is the principal mechanism involved in the protective anti-inflammatory effects of baicalein in $P$. aeruginosa infection.

Together, the results of our study indicate that baicalein, which has both antibacterial and anti-inflammatory effects, can effectively ameliorate tissue injury and slow the progression of $P$. aeruginosa-induced disease by interrupting the vicious cycle of infection and inflammation. Because baicalein possesses novel anti-QS activity and a notable anti-inflammatory effect, it may be broadly applicable against $P$. aeruginosa infection.

\section{Acknowledgments}

This work was supported by the National Natural Science Foundation of China (81260663 and 81260002), the Program for the Cultivation of "139" High-Level Backbone Medical Talents in Guangxi Province (2014GWKJF14), and the Innovation Project of Guangxi Graduate Education (YCBZ2013014). The funders had no role in the study design, data collection and analysis, decision to publish, or preparation of the manuscript. We also thank Dr Liang Yang (Nanyang Technological University, Singapore) for 
generously donating the $P$. aeruginosa PAO1 wild-type strain and its mutants deficient in lasR-rhlR ( $\Delta l a s I-\Delta r h l I)$.

\section{Disclosure}

The authors report no conflicts of interest in this work.

\section{References}

1. Chastre J, Fagon J. Ventilator-associated pneumonia. Am J Respir Crit Care Med. 2002;165(7):867-903.

2. de Oliveira Costa P, Atta EH, da Silva AR. Predictors of 7- and 30-day mortality in pediatric intensive care unit patients with cancer and hematologic malignancy infected with Gram-negative bacteria. Braz J Infect Dis. 2014;18(6):591-599.

3. Banerjee D, Khair OA, Honeybourne D. Impact of sputum bacteria on airway inflammation and health status in clinical stable COPD. Eur Respir J. 2004;23(5):685-691.

4. Hauser AR, Jain M, Bar-Meir M, McColley SA. Clinical significance of microbial infection and adaptation in cystic fibrosis. Clin Microbiol Rev. 2011;24(1):29-70.

5. Wahman S, Emara M, Shawky RM, El-Domany RA, Aboulwafa MM. Inhibition of quorum sensing-mediated biofilm formation in Pseudomonas aeruginosa by a locally isolated Bacillus cereus. J Basic Microbiol. Epub 2015 Aug 19.

6. Pearson JP, Van Delden C, Iglewski BH. Active efflux and diffusion are involved in transport of Pseudomonas aeruginosa cell-to-cell signals. J Bacteriol. 1999;181(4):1203-1210.

7. Rasmussen TB, Givskov M. Quorum sensing inhibitors: a bargain of effects. Microbiology. 2006;152(Pt 4):895-904.

8. Senturk S, Ulusoy S, Bosgelmez-Tinaz G, Yagci A. Quorum sensing and virulence of Pseudomonas aeruginosa during urinary tract infections. J Infect Dev Ctries. 2012;6(6):501-507.

9. Veesenmeyer JL, Hauser AR, Lisboa T, Rello J. Pseudomonas aeruginosa virulence and therapy: evolving translational strategies. Crit Care Med. 2009;37(5):1777-1786.

10. Klausen M, Heydorn A, Ragas P, et al. Biofilm formation by Pseudomonas aeruginosa wild type, flagella and type IV pili mutants. Mol Microbiol. 2003;48(6):1511-1524.

11. Döring G, Conway SP, Heijerman HG, et al. Antibiotic therapy against Pseudomonas aeruginosa in cystic fibrosis: a European consensus. Eur Respir J. 2000;16(4):749-767.

12. Rasko DA, Sperandio V. Anti-virulence strategies to combat bacteriamediated disease. Nat Rev Drug Discov. 2010;9(2):117-128.

13. Cohen TS, Prince AS. Activation of inflammasome signaling mediates pathology of acute $P$. aeruginosa pneumonia. J Clin Invest. 2013; 123(4):1630-1637.

14. Luna CM, Baquero S, Gando S. Experimental severe Pseudomonas aeruginosa pneumonia and antibiotic therapy in piglets receiving mechanical ventilation. Chest. 2007;132(2):523-531.

15. Lee J, Shin H, Kim Y,Paek S, Jin S, Ha U.Pseudomonas aeruginosa-induced IL-1 $\beta$ production is inhibited by Sophora flavescens via the NF- $\mathrm{KB}$ / inflammasome pathways. J Microbiol. 2014;52(12):1044-1049.

16. Sadlon AE, Lamson DW. Immune-modifying and antimicrobial effects of eucalyptus oil and simple inhalation devices. Altern Med Rev. 2010;15(1):33-47.

17. Chaudhary A, Harminder AK, Singh V. A review on the taxonomy, ethnobotany, chemistry and pharmacology of Oroxylum indicum Vent. Indian J Pharm Sci. 2011;73(5):483-490.

18. Zhang H, Chen Q, Zhou W, et al. Chinese medicine injection shuanghuanglian for treatment of acute upper respiratory tract infection: a systematic review of randomized controlled trials. Evid Based Complement Alternat Med. 2013;2013:987326.

19. Cao Y, Dai B, Wang Y, et al. In vitro activity of baicalein against Candida albicans biofilms. Int J Antimicrob Agents. 2008;32(1): 73-77.
20. Wu JA, Attele AS, Zhang L, Yuan C. Anti-HIV activity of medicinal herbs: usage and potential development. Am J Chin Med. 2001;29(1): 69-81.

21. Chen $\mathrm{H}, \mathrm{Gao} \mathrm{Y}, \mathrm{Wu}$ J, et al. Exploring therapeutic potentials of baicalin and its aglycone baicalein for hematological malignancies. Cancer Lett. 2014;354(1):5-11.

22. Sahu BD, Kumar JM, Sistla R. Baicalein, a bioflavonoid, prevents cisplatin-induced acute kidney injury by up-regulating antioxidant defenses and down-regulating the MAPKs and NF-KB pathways. PLoS One. 2015;10(7): 0134139.

23. Zhang Z, Cui W, Li G, et al. Baicalein protects against 6-OHDAinduced neurotoxicity through activation of Keap1/Nrf2/HO-1 and involving PKC $\alpha$ and PI3K/AKT signaling pathways. J Agric Food Chem. 2012;60(33):8171-8182.

24. Fujita M, Shiota S, Kuroda T, et al. Remarkable synergies between baicalein and tetracycline, and baicalein and $\beta$-lactams against methicillin-resistant Staphylococcus aureus. Microbiol Immunol. 2005;49(4):391-396.

25. Zeng Z, Qian L, Cao L, et al. Virtual screening for novel quorum sensing inhibitors to eradicate biofilm formation of Pseudomonas aeruginosa. Appl Microbiol Biotechnol. 2008;79(1):119-126.

26. Bala A, Kumar R, Harjai K. Inhibition of quorum sensing in Pseudomonas aeruginosa by azithromycin and its effectiveness in urinary tract infections. J Med Microbiol. 2011;60(3):300-306.

27. Essar DW, Eberly L, Hadero A, Crawford IP. Identification and characterization of genes for a second anthranilate synthase in Pseudomonas aeruginosa: interchangeability of the two anthranilate synthases and evolutionary implications. J Bacteriol. 1990;172(2):884-900.

28. Hentzer M, Riedel K, Rasmussen TB, et al. Inhibition of quorum sensing in Pseudomonas aeruginosa biofilm bacteria by a halogenated furanone compound. Microbiology. 2002;148(Pt 1):87-102.

29. Ohman DE, Cryz SJ, Iglewski BH. Isolation and characterization of Pseudomonas aeruginosa $\mathrm{PAO}$ mutant that produces altered elastase. J Bacteriol. 1980;142(3):836-842.

30. Pham TH, Webb JS, Rehm BH. The role of polyhydroxyalkanoate biosynthesis by Pseudomonas aeruginosa in rhamnolipid and alginate production as well as stress tolerance and biofilm formation. Microbiology. 2004;150(Pt 10):3405-3413.

31. Wu H, Lee B, Yang L, et al. Effects of ginseng on Pseudomonas aeruginosa motility and biofilm formation. FEMS Immunol Med Microbiol. 2011;62(1):49-56.

32. Alipour M, Suntres ZE, Lafrenie RM, Omri A. Attenuation of Pseudomonas aeruginosa virulence factors and biofilms by coencapsulation of bismuth-ethanedithiol with tobramycin in liposomes. J Antimicrob Chemother. 2010;65(4):684-693.

33. Gooderham WJ, Bains M, McPhee JB, Wiegand I, Hancock RE. Induction by cationic antimicrobial peptides and involvement in intrinsic polymyxin and antimicrobial peptide resistance, biofilm formation, and swarming motility of PsrA in Pseudomonas aeruginosa. J Bacteriol. 2008;190(16):5624-5634.

34. Franklin MJ, Ohman DE. Identification of algI and algJ in the Pseudomonas aeruginosa alginate biosynthetic gene cluster which are required for alginate $\mathrm{O}$ acetylation. J Bacteriol. 1996;178(8):2186-2195.

35. Knutson CA, Jeanes A. A new modification of the carbazole analysis: application to heteropolysaccharides. Anal Biochem. 1968;24(3): 470-481.

36. Sarabhai S, Harjai K, Sharma P, Capalash N. Ellagic acid derivatives from Terminalia chebula Retz increase the susceptibility of Pseudomonas aeruginosa to stress by inhibiting polyphosphate kinase. $J$ Appl Microbiol. 2015;118(4):817-825.

37. Makemson J, Eberhard A, Mathee K. Simple electrospray mass spectrometry detection of acylhomoserine lactones. Luminescence. 2006; 21(1):1-6.

38. Ortega-González M, Sánchez de Medina F, Molina-Santiago C, et al. Fructooligosacharides reduce Pseudomonas aeruginosa PAO1 pathogenicity through distinct mechanisms. PLoS One. 2014;9(1):e85772. 
39. Singh S, Barr H, Liu YC, et al. Granulocyte-macrophage colony stimulatory factor enhances the pro-inflammatory response of interferon$\gamma$-treated macrophages to Pseudomonas aeruginosa infection. PLoS One. 2015;10(2):e0117447.

40. Zhao Z, Yan S, Yu Y, et al. An aqueous extract of Yunnan baiyao inhibits the quorum-sensing-related virulence of Pseudomonas aeruginosa. J Microbiol. 2013;51(2):207-212.

41. Rashid MH, Kornberg A. Inorganic polyphosphate is needed for swimming, swarming, and twitching motilities of Pseudomonas aeruginosa Proc Natl Acad Sci U S A. 2000;97(9):4885-4890.

42. Rasamiravaka T, Labtani Q, Duez P, El Jaziri M. The formation of biofilms by Pseudomonas aeruginosa: a review of the natural and synthetic compounds interfering with control mechanisms. BioMed Res Int. 2015;2015:759348.

43. Epelman S, Stack D, Bell C, et al. Different domains of Pseudomonas aeruginosa exoenzyme S activate distinct TLRs. J Immunol. 2004; 173(3):2031-2040.

44. Trinchieri G, Sher A. Cooperation of Toll-like receptor signals in innate immune defence. Nat Rev Immunol. 2007;7(3):179-190.

45. Pollard AJ, Currie A, Rosenberger CM, Heale J, Finlay BB, Speert DP. Differential post-transcriptional activation of human phagocytes by different Pseudomonas aeruginosa isolates. Cell Microbiol. 2004;6(7): 639-650.

46. Wang W, Zhou P, Xu C, Zhou X, Hu W, Zhang J. Baicalein attenuates renal fibrosis by inhibiting inflammation via down-regulating NF- $\kappa \mathrm{B}$ and MAPK signal pathways. J Mol Histol. 2015;46(3):283-290.

47. Gómez-Garcés JL, Gil-Romero Y, Sanz-Rodríguez N, Muñoz-Paraíso C, Regodón-Domínguez M. [In vitro activity of fosfomycin, alone or in combination, against clinical isolates of carbapenem resistant Pseudomonas aeruginosa] Actividad in-vitro de fosfomicina, sola o en combinaciones, frente a aislamientos clínicos de Pseudomonas aeruginosa resistentes a carbapenémicos. Enferm Infecc Microbiol Clin. Epub 2015 Aug 12. Spanish.

48. Hentzer M, Wu H, Andersen JB. Attenuation of Pseudomonas aeruginosa virulence by quorum sensing inhibitors. EMBO J. 2003;22(15): 3803-3815.
49. Rasmussen TB, Bjarnsholt T, Skindersoe ME, et al. Screening for quorum-sensing inhibitors (QSI) by use of a novel genetic system, the QSI selector. J Bacteriol. 2005;187(5):1799-1814.

50. Rasmussen TB, Skindersoe ME, Bjarnsholt T. Identity and effects of quorum-sensing inhibitors produced by Penicillium species. Microbiology. 2005;151(Pt 5):1325-1340.

51. Stehling EG, da Silveira WD, da Silva Leite D. Study of biological characteristics of Pseudomonas aeruginosa strains isolated from patients with cystic fibrosis and from patients with extra-pulmonary infections. Braz J Infect Dis. 2008;12(1):86-88.

52. Lau GW, Hassett DJ, Ran H, Kong F. The role of pyocyanin in Pseudomonas aeruginosa infection. Trends Mol Med. 2004;10(12):599-606.

53. O’May C, Tufenkji N. The swarming motility of Pseudomonas aeruginosa is blocked by cranberry proanthocyanidins and other tannincontaining materials. Appl Environ Microb. 2011;77(9):3061-3067.

54. Davies DG, Parsek MR, Pearson JP, Iglewski BH, Costerton JW, Greenberg EP. The involvement of cell-to-cell signals in the development of a bacterial biofilm. Science. 1998;280(5361):295-298.

55. Glessner A, Smith RS, Iglewski BH, Robinson JB. Roles of Pseudomonas aeruginosa las and rhl quorum-sensing systems in control of twitching motility. J Bacteriol. 1999;181(5):1623-1629.

56. Lappalainen U, Whitsett JA, Wert SE, Tichelaar JW, Bry K. Interleukin- $1 \beta$ causes pulmonary inflammation, emphysema, and airway remodeling in the adult murine lung. Am J Respir Cell Mol Biol. 2005; 32(4):311-318.

57. Medzhitov R, Janeway CJ Jr. Innate immune recognition: mechanisms and pathways. Immunol Rev. 2000;173(1):89-97.

58. Girardin E, Grau GE, Dayer J, Roux-Lombard P, Lambert P. Tumor necrosis factor and interleukin-1 in the serum of children with severe infectious purpura. N Engl J Med. 1988;319(7):397-400.

59. He X, Wei Z, Zhou E, et al. Baicalein attenuates inflammatory responses by suppressing TLR4 mediated NF- $\kappa B$ and MAPK signaling pathways in LPS-induced mastitis in mice. Int Immunopharmacol. $2015 ; 28(1): 470-476$. 


\section{Supplementary materials}

Table SI Primers used in this study for real-time polymerase chain reaction

\begin{tabular}{ll}
\hline Target gene & Primer sequences \\
\hline I6S ribosomal RNA (PA 5369.5) & Forward: CGTCCGGAAACGGCCGCT \\
lasl (PA 1432) & Reverse: CTCTCAGACCAGTTACGG \\
& Forward: AGGCGTGGAGAAGATGATG \\
lasR (PA 1430) & Reverse: ATCTGGGTCTTGGCATTGAG \\
& Forward: GTGGAAAATTGGAGTGGAGCG \\
rhll (PA 3476) & Reverse: GTAGTTGCCGACGATGAAGG \\
& Forward: TGCAGCCATTCCGGGTGGTA \\
rhIR (PA 3477) & Reverse: TCGTTCGCAACGGCGTGAT \\
& Forward: GGGTTGGACATCAGCATCGG \\
& Reverse: TTGCTCAGCGTGCTTTCCGT \\
\hline
\end{tabular}

A OdDHL
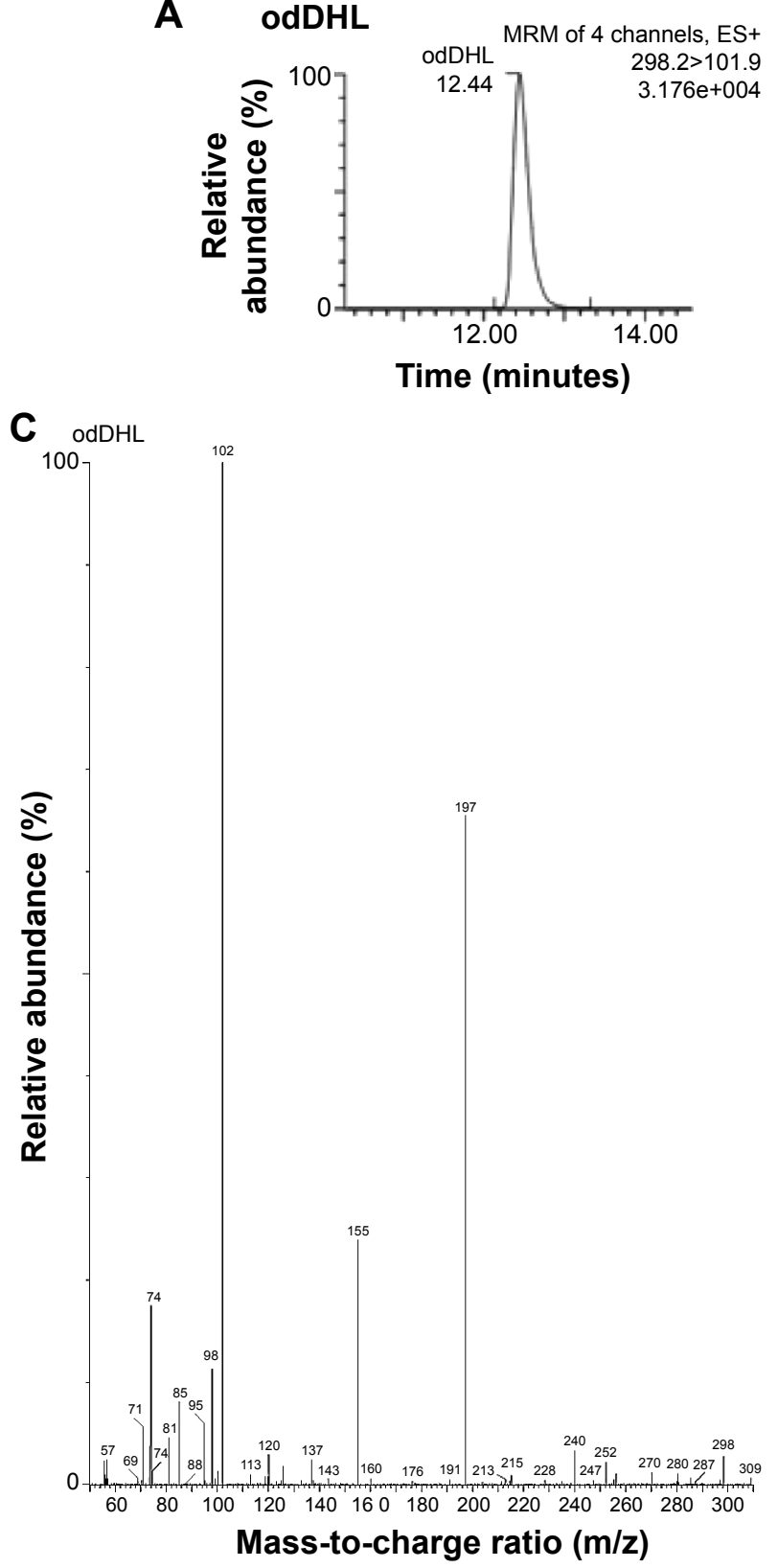

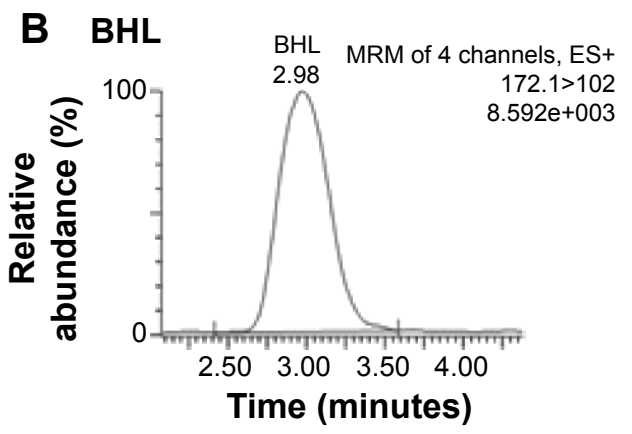

D

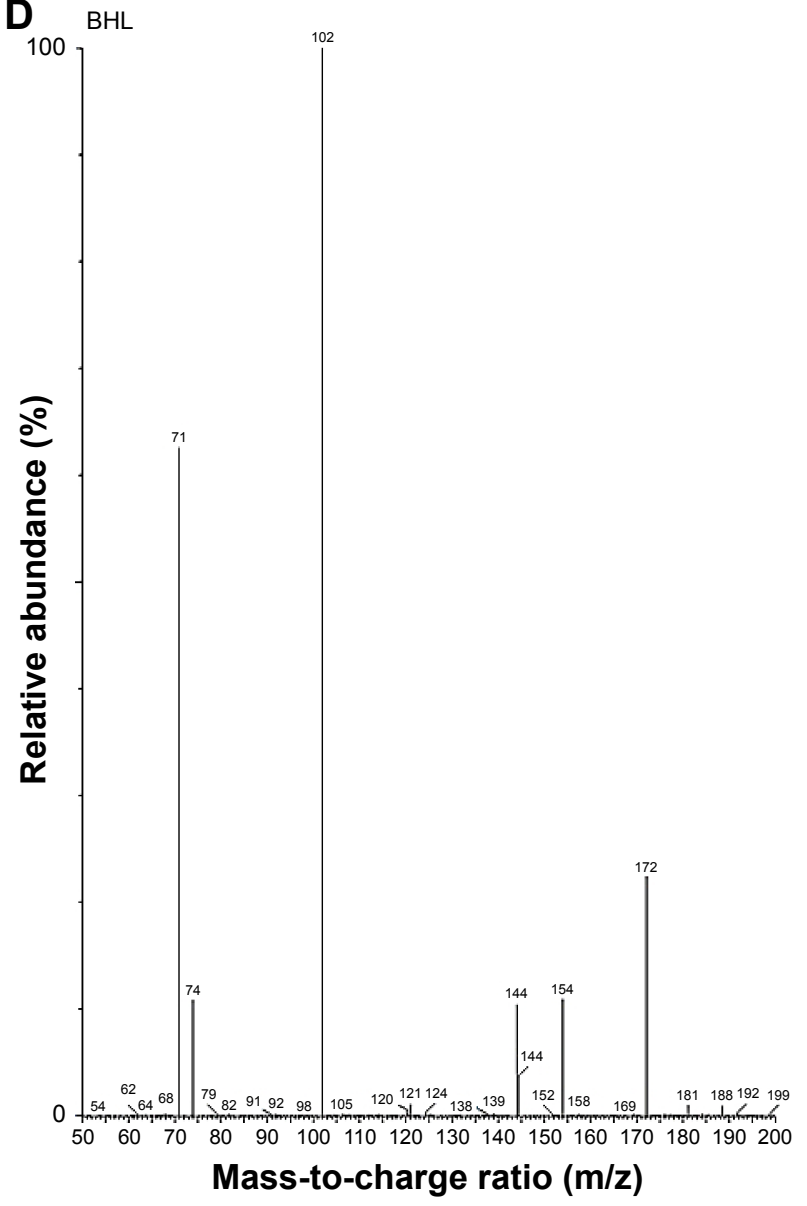

Figure SI (Continued) 
E Compound name: odDHL

Correlation coefficient: $r=0.999496, r^{2}=0.998992$

Calibration curve: $5.58616^{*} x+2,376.32$

Response type: external std, area

Curve type: linear, Origin: exclude

Weighting: null, Axis trans: none

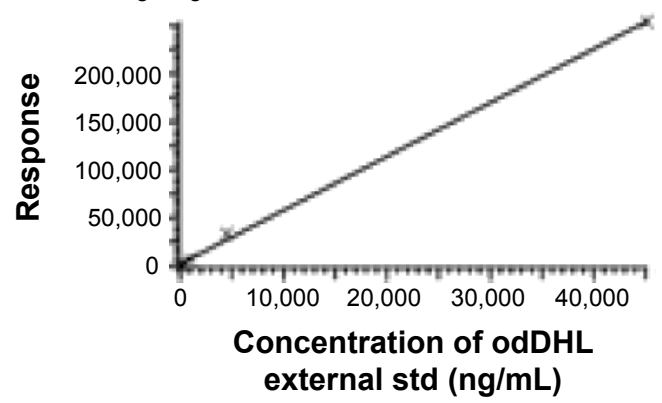

G Control

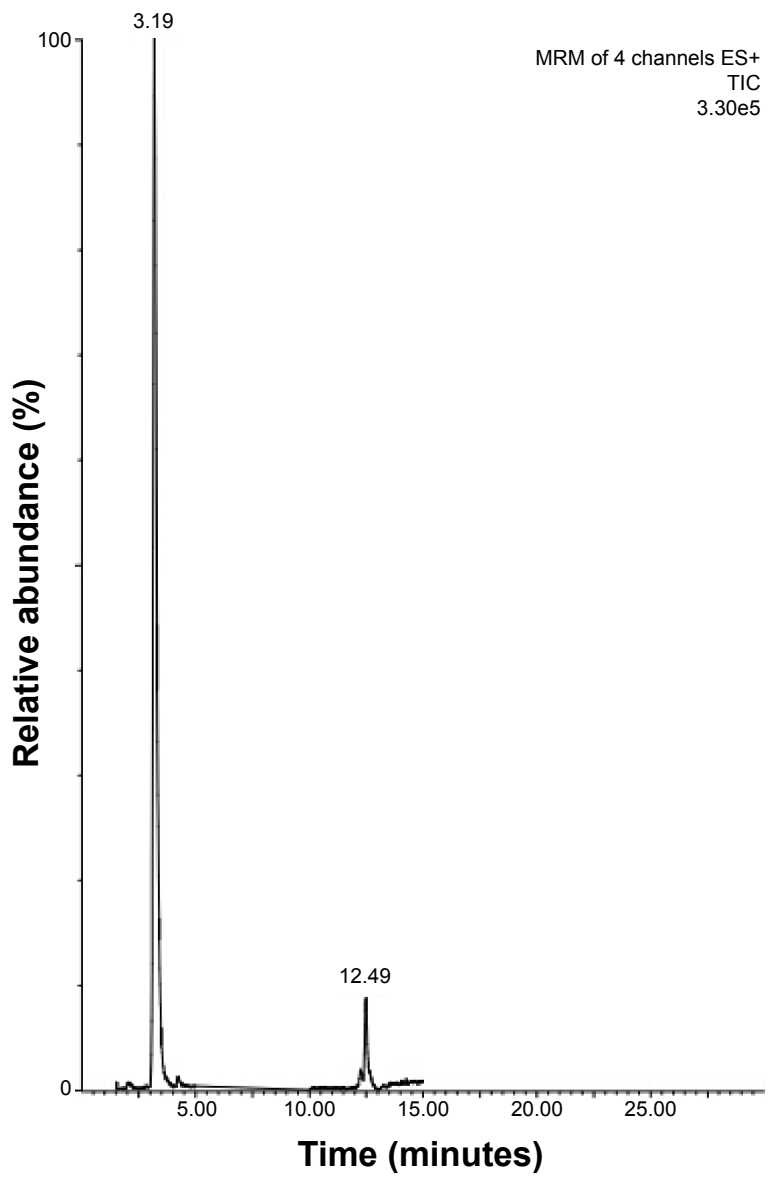

F Compound name: $\mathrm{BHL}$

Correlation coefficient: $r=0.998393, r^{2}=0.996789$

Calibration curve: $1.64156^{*} x+1,446.24$

Response type: external std, area

Curve type: linear, Origin: exclude

Weighting: null, Axis trans: none

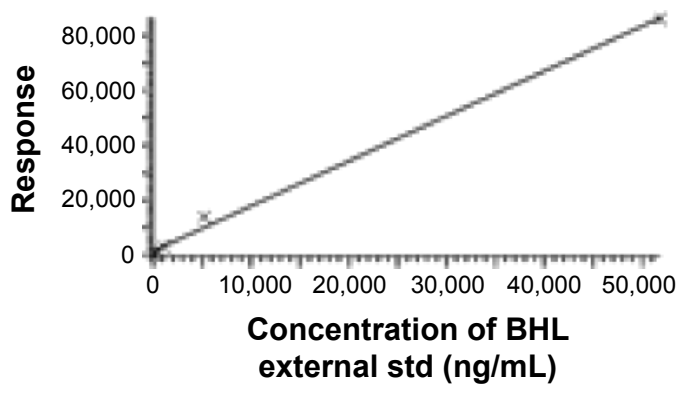

H $128 \mu \mathrm{gg} / \mathrm{mL}$

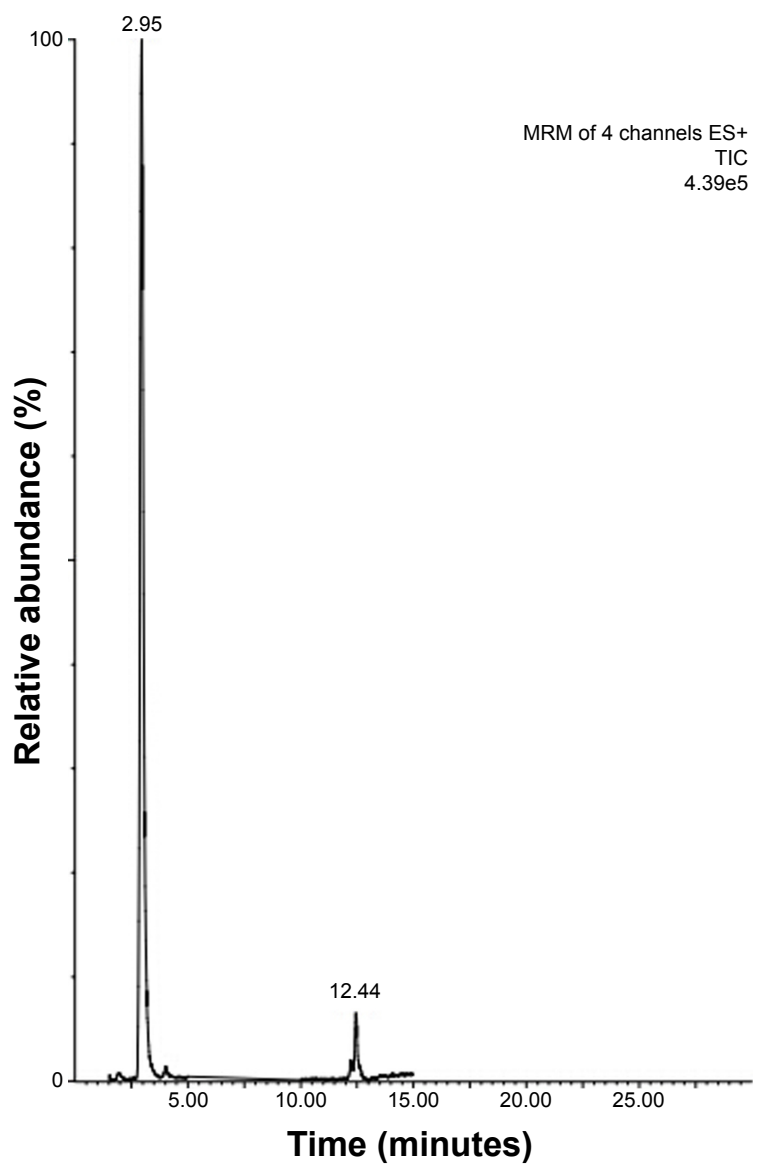

Figure SI (Continued) 

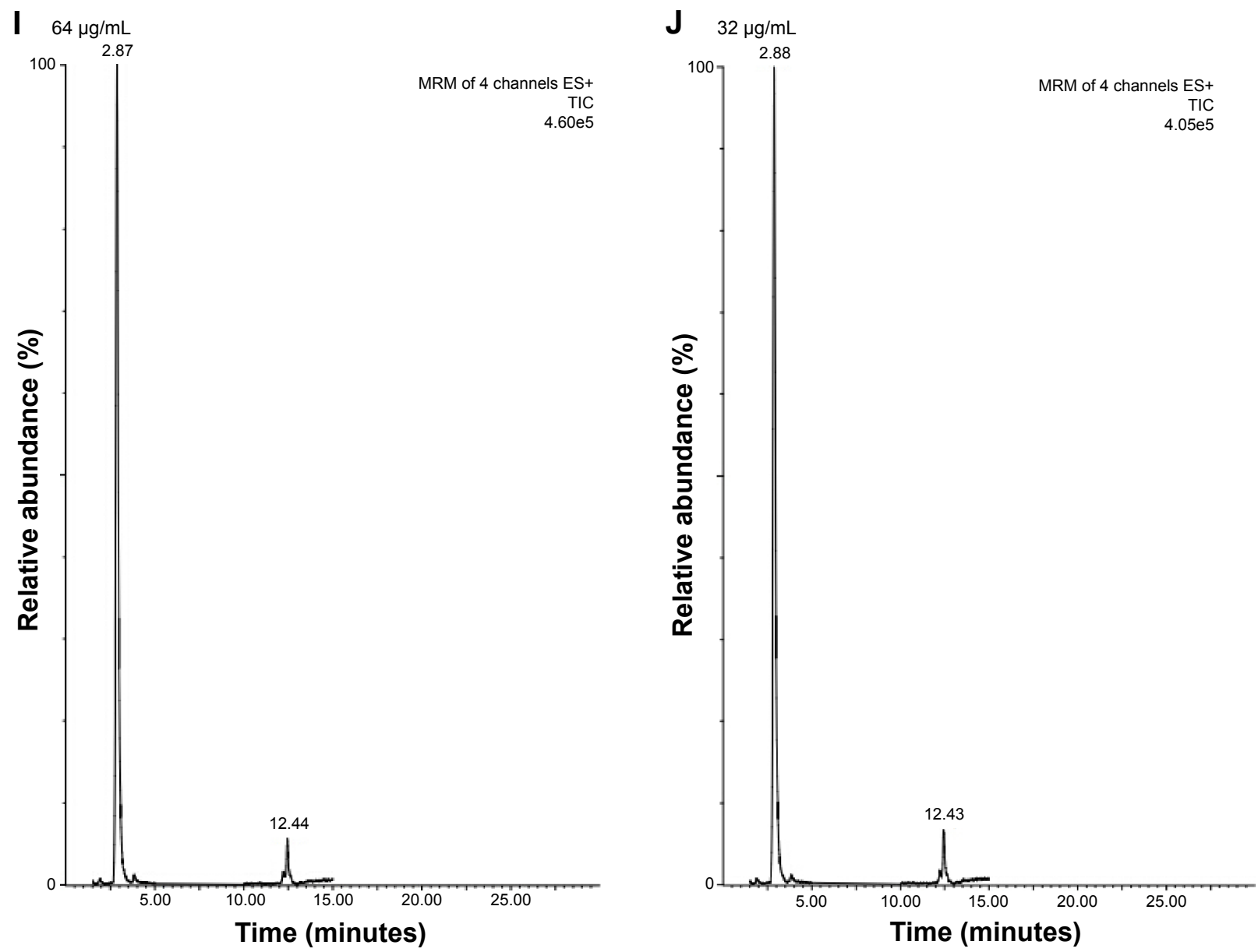

Figure SI HPLC-MS analysis of AHLs of Pseudomonas aeruginosa.

Notes: The retention times of odDHL (A) and BHL (B) were detected by HPLC, and the MS/MS fragment peaks of odDHL (C) and BHL (D) were analyzed by ESIMS. Standard curves of odDHL (E) and BHL (F) generated from the pure compounds were drawn for quantification. Drug-free control sample (G) and treatment with $128 \mu \mathrm{g} / \mathrm{mL}(\mathbf{H}), 64 \mu \mathrm{g} / \mathrm{mL}(\mathbf{I})$, and $32 \mu \mathrm{g} / \mathrm{mL}(\mathrm{J})$ baicalein samples were quantitated by HPLC-MS in MRM mode, respectively.

Abbreviations: HPLC, high-performance liquid chromatography; MS, mass spectrometry; odDHL, N-(3-oxododecanoyl)-L-homoserine lactone; BHL, N-butanoyl-Lhomoserine lactone; ESI, electrospray ionization; MRM, multiple reaction monitoring; ES, electron spray; TIC, total iron count; std, standard; ES+, positive ionization mode of the electron spray.

Table S2 Precursor ions $(M+H)^{+}$and fragmentation ions derived from the acyl-chain moiety $(M+H-I 0 I)^{+}$of $A H L s$ detected and identified by HPLC-MS

\begin{tabular}{llll}
\hline AHLs & Retention time (minutes) & $(\mathbf{M}+\mathbf{H})^{+}$ions $\mathbf{( m / z )}$ & Fragmentation ions $(\mathbf{m} / \mathbf{z})$ \\
\hline OdDHL & 12.44 & 298 & 197 \\
BHL & 3.02 & 172 & 71 \\
\hline
\end{tabular}

Abbreviations: AHLs, N-acyl-L-homoserine lactones; HPLS, high-performance liquid chromatography; MS, mass spectrometry; odDHL, N-(3-oxododecanoyl)-L-homoserine lactone; BHL, N-butanoyl-L-homoserine lactone. 


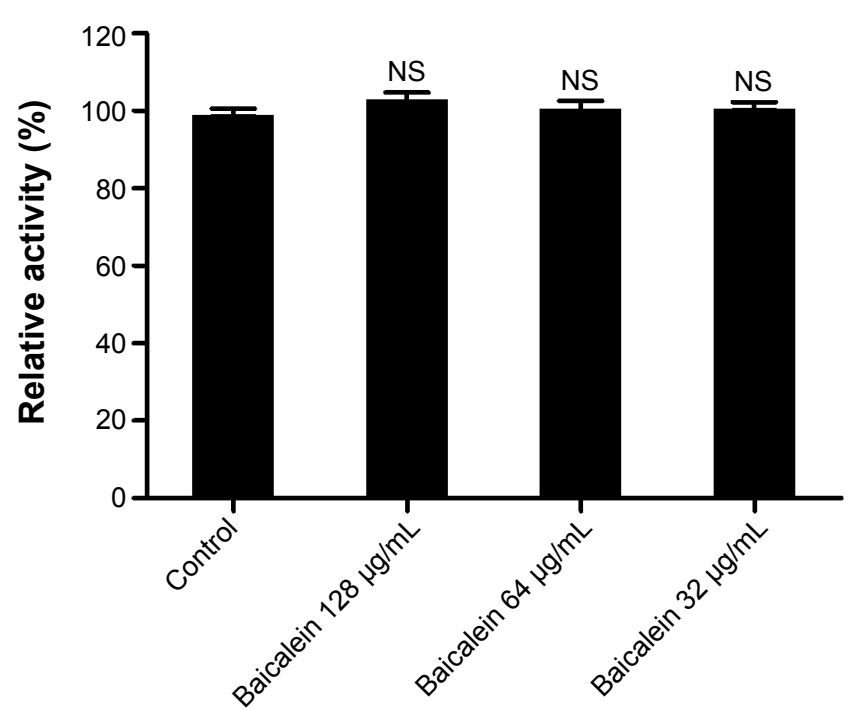

Figure S2 Effects of different sub-minimum inhibitory concentration levels of baicalein on the viability of primary macrophages evaluated using the MTT assay. Note: Data represent mean \pm standard error of three independent experiments.

Abbreviations: MTT, 3-(4,5-dimethylthiazol-2-yl)-2,5-diphenyltetrazolium bromide; NS, not significant.

\section{Publish your work in this journal}

Drug Design, Development and Therapy is an international, peerreviewed open-access journal that spans the spectrum of drug design and development through to clinical applications. Clinical outcomes, patient safety, and programs for the development and effective, safe, and sustained use of medicines are a feature of the journal, which has also been accepted for indexing on PubMed Central. The manuscript management system is completely online and includes a very quick and fair peer-review system, which is all easy to use. Visit http://www.dovepress.com/testimonials.php to read real quotes from published authors.

\footnotetext{
Submit your manuscript here: http://www.dovepress.com/drug-design-development-and-therapy-journal
} 